\title{
A Spatially Resolved and Quantitative Model of Early Atherosclerosis
}

\author{
Moritz P. Thon • Mary R. Myerscough • \\ Michael W. Gee
}

Received: date / Accepted: date

\begin{abstract}
Atherosclerosis is a major burden for all societies and there is a great need for a deeper understanding of involved key inflammatory, immunological and biomechanical processes. A decisive step for the prevention and medical treatment of atherosclerosis is to predict what conditions determine whether early atherosclerotic plaques continue to grow, stagnate or become regressive. The driving biological and mechanobiological mechanisms that determine the stability of plaques are yet not fully understood.

We develop a spatially resolved and quantitative mathematical model of key contributors of early atherosclerosis. The stability of atherosclerotic model plaques is assessed to identify and classify progression-prone and progressionresistant atherosclerotic regions based on measurable or computable in vivo inputs, such as blood cholesterol concentrations and wall shear stresses. The model combines Darcy's law for the transmural flow through vessels walls, the Kedem-Katchalsky equations for endothelial fluxes of lipoproteins, a quantitative model of early plaque formation from a recent publication and a novel submodel for macrophage recruitment.

The parameterization and analysis of the model suggest that the advective flux of lipoproteins through the endothelium is decisive, while the influence of the advective transport within the artery wall is negligible. Further, regions in arteries with an approximate wall shear stress exposure below $20 \%$ of the average exposure and their surroundings are potential regions where progressionprone atherosclerotic plaques develop.
\end{abstract}

Moritz P. Thon · Michael W. Gee (凶)

Mechanics \& High Performance Computing Group, Technical University of Munich, Parkring 35, 85748 Garching b. München, Germany

E-mail: gee@tum.de

Mary R. Myerscough

School of Mathematics and Statistics F07, University of Sydney, New South Wales 2006, Australia 
Keywords atherosclerosis · parameter estimation · stability analysis · macrophage recruitment $\cdot$ transmural flow $\cdot$ lipoprotein flux

\section{Introduction}

Atherosclerosis is a chronic inflammatory vascular disease of the artery wall 78 56. 24. It causes a pathological alteration of the intima and adjacent media, and is characterized by the development of non-resolving inflammatory lesions called atherosclerotic plaques. Atherosclerosis causes severe cardiovascular sequelae like stroke or myocardial infarction and so is a major contributor to worldwide morbidity, loss of productive life years and mortality [66, 102].

In this contribution, we present a spatially resolved and quantitative mathematical model that allows us to investigate the influence of spatial effects, such as diffusion, advection and inhomogeneous distributions on atherosclerosis progression. We use the model to assess the long-term stability of early atherosclerotic plaques based on measurable or computable in vivo inputs. Due to the availability of the required inputs and the great need of predictive models of atherosclerosis, this model is a significant step toward mathematical and computational models that allow to predict the stability of early plaques in a clinical setting.

A significant first step for the onset of the inflammatory atherosclerotic processes is a dysfunction of the endothelium lining the vessel wall which increases the penetration of low-density lipoproteins (LDL) from the bloodstream into the vessel wall. Over the past decades, more and more evidence has been found that hemodynamical factors, such as low wall shear stresses (WSS), resulting from flow recirculations and oscillatory blood flows, locally disturb the endothelium in a manner that increases the LDL penetration [72,6,53, 91 .

LDL and high-density lipoproteins (HDL) retained in the artery wall are prone to oxidative modifications by endothelial cells and macrophages [84, 35, 51, 67. The modification of native LDL (i.e., LDL as contained in blood) renders it pro-inflammatory 100,84,101 and initiates an immune response in the artery wall. Modified LDL activates endothelial cells causing the production of vascular cell adhesion molecule-1 (VCAM-1) and other cell adhesion molecules [17, 31, which increases the adhesiveness of the endothelium with respect to monocytes [4,54,42,41].

Monocytes in the bloodstream roll and adhere on the endothelium surface due to the presence of VCAM-1 [2,55], which enables their migration into the artery wall. Monocytes, however, are also detached by the mechanical drag force of the fluid, i.e., the WSS acting on the endothelium [42,2]. When modified LDL is present, endothelial cells become activated and increase VCAM1 production [17] which enhances the binding of monocytes and reduces the amount of WSS induced detachment 42,2. Moreover, modified LDL causes the production of monocyte chemoattractant protein-1 (MCP-1) by endothelial cells and smooth muscle cells in the adventitia creating a chemotactic gradient of MCP-1 within the artery wall 33] that causes firmly attached monocytes to 
actively migrate through the endothelium into the intima [55]. Once inside the intima, monocytes may continue to transmigrate into the media [60, 80] due to chemotactic gradients or differentiate into macrophages [21,55].

Monocyte-derived macrophages are at the crux of early atherogenesis as they populate inflamed tissue, actively scavenge and ingest modified LDL, undergo controlled cell death (apoptosis) and consume other dead and dying macrophages (efferocytosis) [65, 32, 33, 65]. Macrophages store the cholesterol from ingested LDL as intracellular free and esterified cholesterols, which are at a dynamic buffer-like equilibrium [7,8,92]. The maintenance of intracellular cholesterol balance between essential and cytotoxic limits is disrupted in plaque macrophages when there is an excess consumption of modified LDL [49] and poor access to HDL which exports intracellular free cholesterol out of cells [7] 73. Above the cytotoxic cholesterol limit macrophage necrosis (uncontrolled cell death) is inevitable $[57,26,88$. This causes the accumulation of necrotic debris and extracellular lipids that form the necrotic core inside advanced plaques 65 , 32. Thus, cholesterol-disrupted macrophages can induce a progression of the disease from its asymptomatic early stages (type I - IIb in the classification by the American Heart Association [82]) to potentially symptomatic and threatening advanced stages (type III or higher).

Mathematical and computational models have an important role to play in increasing the understanding of atherosclerosis. A broad spectrum of models of various aspects of atherosclerosis has been created over recent decades. A comprehensive tabular overview of existing models of atherosclerosis and their specific applications is given in the review by Parton et al. [71]. An overview of models of the underlying cardiovascular mechanics and their connection to atherosclerosis is given in the Thesis by Thon [91].

The transport of LDL through the endothelium is well-studied and commonly assessed by the equations of Kedem and Katchalsky [45, 103, 10, 43, 74, 93 90. The crucial role of biomechanics in the penetration of LDL is usually incorporated by a WSS-dependent adaption of the Kedem-Katchalsky equations. To this end, some researchers model the different LDL transport pathways in full detail and estimate their individual alteration based on local WSS 69, 70]. In contrast, other approaches consider only a WSS-dependent hydraulic conductivity [85] or utilize heuristic laws that represent changes in the diffusive permeability [10,93. These models drastically reduce the complexity but still mimic a physiologically correct behavior. Nevertheless, the interplay of hemodynamic forces, endothelial permeability and atherosclerosis progression is not yet understood in full detail [72,77, 97, 91].

Opinion is divided on the relative importance of different driving mechanisms for LDL transport into and within the intima. According to the KedemKatchalsky equations, the flux through the endothelium is a superposition of a concentration gradient-driven diffusive flux and an advective flux. The advective flux is induced by the transmural flow of blood plasma driven by the pressure gradient across the endothelium. However, there is no experimental data that allow to determine the importance of the advective flux. This applies particularly to the LDL transport within the artery wall which might be driven 
by both diffusion and advection, or solely by diffusion. Many researchers model the transmural flow across the endothelium and within the artery wall using porous media approaches $[74,94,103,70]$. Others model the artery wall as an elastic solid and the LDL transport as a purely diffusive process [19, 64, 27, 10 95. 93. The importance of the transmural flow on the endothelial and intimal lipoprotein transport and on early atherosclerosis is controversial [95, 70] and is thus addressed in this contribution.

In contrast to the transport of LDL, the penetration of HDL and the recruitment of macrophages are key aspects of atherosclerosis that have received scant attention [83, 15, 30]. Due to the related structure and size of LDL and HDL [46,75], however, we assume that HDL will have transport mechanisms that are analogous to LDL 83 . The lack of modeling of macrophage recruitment is problematic as the active migration and movement of monocytes is quite distinct from lipoprotein transport. Only very limited work has been done on modeling of monocyte recruitment as a function of the current state of a plaque in general [15,9] and the activation of the endothelium in particular. Due to poor availability of quantitative experimental data, the modeling and quantification of macrophage recruitment remained a challenge.

The formation of early atherosclerotic plaques is modeled and quantified in a previous work by the present authors [92. In that publication, a parameterized set of ordinary differential equations (ODEs) was developed that predicts key inflammatory and lipid processes of early atherosclerosis: LDL modification by macrophages and endothelial cells, HDL protection against LDL modification, LDL ingestion by macrophages, intracellular cholesterol cycle within macrophages, reverse cholesterol transport to HDL as well as macrophage apoptosis. The established ODE model is able to predict the stability of early model plaques based on the recruitment of LDL, HDL and macrophages. However, the recruitment rates were a priori specified constants that do not change as the plaque progresses and cannot be estimated from measurable or computable in vivo inputs in a straightforward manner. It was shown, in particular, that the rate of macrophage recruitment is crucial for the stability of early plaques and plaque classification into progression-prone and progression-resistant plaques. In addition, the model neglects all spatial aspects, which is reasonable only for in vitro experiments. Spatial effects, such as the diffusivity of species, their transport with the transmural flow or their locally varying recruitment into the artery wall due inhomogeneous WSS patterns can have a severe impact on the local disease progression in vivo.

In this contribution, we focus on the modeling of the endothelial penetration of species and their transmural transport within the artery wall. The explicit modeling of the endothelial lipoprotein and macrophage transport mechanisms allows us to estimate recruitment rates and make predictions about plaque progression based on three measurable or computable physiological in vivo inputs: the LDL and HDL blood cholesterol concentrations and the WSS that the plaque is exposed to. The quantitative modeling of local lipoprotein concentrations in blood and local WSS has been frequently addressed, see [93, 
70, 47, 19,58, and therein. In this work, these concentrations and WSS are assumed to be known model inputs.

The spatially resolved model presented here is based on basic continuum mechanical laws and naturally brings together submodels which describe the penetration of key species into the artery wall as well as their transport with the transmural flow, inflammatory processes and lipid dynamics within the artery wall. While previous models for atherosclerosis also considered some of theses processes [10,15, 16, 30, 47, 103], our model is the first to provide a complete predictive parameter set for all those key processes of early atherosclerosis.

A sensitivity analysis of our model with respect to its estimated parameters is performed that identifies crucial parameters and processes. Further, the model's stability is assessed based on the three in vivo inputs in order to identify and classify regions of progression-prone (type IIa) and progressionresistant (type IIb) model plaques [82]. Moreover, the model is used to assess the importance of the diffusive and advective lipoprotein fluxes through the endothelium and within the artery wall. Finally, the influence of locally varying WSS on the model plaque outcome is investigated.

The structure of this work is as follows: In Sect. 2, before the spatially resolved model is established, submodels of the recruitment of macrophages as well as of the fluxes of LDL and HDL are developed. Sect. 3 gives a complete parameter set for the models and all computational results derived from them. The results are discussed and critically assessed in Sect. 4. Supporting details are given in Appendix A and Appendix B

\section{Models}

The spatially resolved, quantitative model of early atherosclerosis we develop in this work builds upon a previous publication [92. In our earlier work we developed and parameterized an ODE model to describe the formation of early atherosclerotic plaque based on several in vitro experiments by [7, 8, 35, $, 51,59$ 104. This ODE model predicts three different plaque outcomes depending on assumed fixed rates of macrophage recruitment, LDL influx and HDL influx which depend on the physiology and diet of the plaque's host, and the position of the plaque within the cardiovascular system. The model estimates temporal changes of the following key species: concentration of native LDL $\ell$, concentration of modified LDL $\tilde{\ell}$, concentration of native HDL $h$, concentration of intracellular free cholesterol $f$, concentration of intracellular cholesterol ester $b$ and density of macrophages $m$.

This work also models the key species above using the same notation, but employs a continuum approach where the concentration of each species is represented as a function of time and space. The time- and space-dependent behavior of the species is modeled by systems of partial differential equations that naturally include spatial effects. A schematic overview of the model's species and their inflammatory, lipid and recruitment processes is given in Figure 1 . 


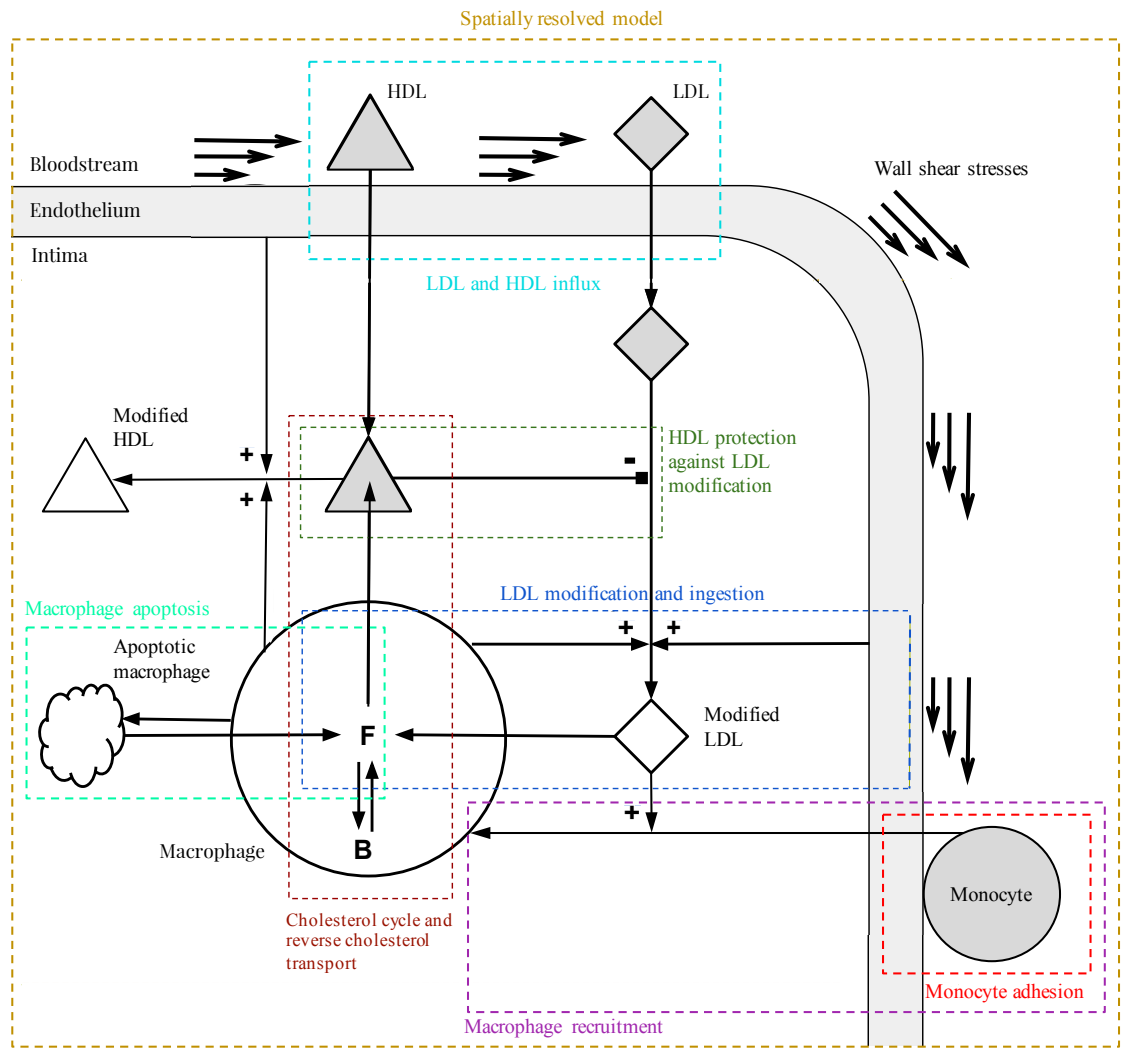

Fig. 1 Overview of key species and their interaction considered in the spatially resolved model. Native LDL (diamond, grey) and HDL (triangle, grey) enter the vessel wall at rates dependent on their blood concentrations and the magnitude of wall shear stress exerted on the endothelium by blood flow. Monocytes (circle, grey) adhere to the endothelium depending on the wall shear stresses and migrate into the vessel wall based on the concentration of modified LDL inside the vessel wall (diamond, white). Migrated monocytes differentiate into macrophages (circle, white), ingest modified LDL, efferocytose apoptotic macrophages (cloud), export free cholesterol to HDL and undergo apoptosis. Free cholesterol in macrophages is converted to esterified cholesterol (B) and stored so that free cholesterol remains between essential and cytotoxic limits. LDL and HDL are oxidatively modified by macrophages and the endothelium but unmodified HDL counteracts LDL oxidation. The processes modeled and quantified in this work are encircled by red, purple and turquoise frames. The remaining colored frames correspond to quantified submodels established in a previous work by the present authors 92. All species inside the vessel wall are subject to transport by diffusion. LDL and HDL are also transported by advection with the transmural flow. 
In the rest of this section, submodels for the recruitment of macrophages and the flux of lipoproteins are first developed and quantified. Next, the spatially resolved model is formulated. This can be subdivided into a model for the transmural flow and a model for the penetration, spatial transport and interaction of key species of early atherosclerosis.

\subsection{Submodels}

In this section, we develop the submodels that describe the recruitment of macrophages and the fluxes of lipoproteins through the endothelium. The submodels are parameterized using existing experimental and theoretical literature. If specific experimental data exist, then we choose relationships that best represent the data.

\subsubsection{Monocyte Adhesion}

Monocytes migrate from the bloodstream into the artery wall at a rate that depends on the local flow condition of blood and the current activation of the endothelium. Our submodel for monocyte adhesion is based on experimental in vitro results by Jeng et al. 42. All parameters in the submodel are either experiment-specific (i.e. are specified by the experimental procedure) or estimated by a least-squares fit to the experimental results.

Experimental setup The adhesion of human monocytes on human vein endothelial cells in the absence of flow and their binding under flow conditions in vitro was investigated by Jeng et al. 42 using a two-stage experimental setup. First, specified concentrations of native LDL $\ell_{0}$ and modified LDL $\tilde{\ell}_{0}$ were incubated with cultured endothelial cell monolayers $e$ on coverslips for specified time periods $T_{\mathrm{Mod}}$. The endothelial cells oxidatively modified native LDL 35, 51] and became activated by the presence of modified LDL which increased their adhesiveness with respect to monocytes $31,2,54$. Subsequently, all LDL was removed and monocytes were added to the endothelial cells for specified time periods $T_{\mathrm{Adh}}$, where they adhered to the endothelial cells. The coverslips were placed in a flow chamber where the flux over the monolayer of cells was increased incrementally, exposing the monocytes to varying WSS levels $\tau$. Among other things, the number of adhered monocytes $m_{\text {Adh }}$ preliminary to the flow chamber experiment and the number of remaining monocytes after each increase of WSS exposure was counted for various experiment-specific values of $\ell_{0}, \tilde{\ell}_{0}, e, T_{\mathrm{Mod}}, T_{\mathrm{Adh}}, \tau$ as shown in Table 1

Mathematical submodel The observations made in the flow chamber experiments in 42 are driven by the modification of native LDL by endothelial cells, the adhesion of monocytes on modified LDL-activated endothelial cells and the detachment of monocytes due to exposure to WSS. As in 92, the modification of native LDL by endothelial cells is described by a linear relationship in 
Table 1 Experiment-specific parameters of the submodel of monocyte adhesion in vitro in analogy to the experimental setups by Jeng et al. [42.

\begin{tabular}{|c|c|c|c|c|c|c|}
\hline Experiment & $\frac{\Xi}{z}$ & 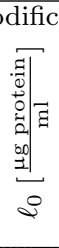 & 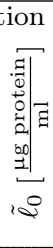 & 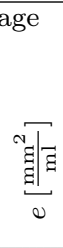 & $\begin{array}{l}\Xi \\
E \\
E \\
E\end{array}$ & 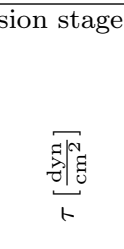 \\
\hline 42, Table $1, \ell_{0}=0, \tilde{\ell}_{0}=0$ & 6 & 0 & 0 & 2.68 & 0.5 & $0-30.52$ \\
\hline 42, Table $1, \ell_{0}=25, \tilde{\ell}_{0}=0$ & 6 & 25 & 0 & 2.68 & 0.5 & $0-30.52$ \\
\hline 42, Table $1, \ell_{0}=0, \tilde{\ell}_{0}=25$ & 6 & 0 & 25 & 2.68 & 0.5 & $0-30.52$ \\
\hline
\end{tabular}

All values are given in the units of the submodel.

their concentrations $\ell$ and $e$ and the initial LDL modification lag phase is accounted for in the model by a reduction of $4 \mathrm{~h}$ of the experimental time period $T_{\text {Mod }}$. The activation of endothelial cells by modified LDL saturates as the concentration of modified LDL $\tilde{\ell}$ increases (see [42], Fig. 5 and [31, Fig. 1). Monocytes adhere on endothelial cells also in the absence of LDL (see [42, Fig. 3 and [31, Fig. 1). Therefore, the activation of endothelial cells is modeled using a saturating kinetic. The WSS-dependent detachment of adhered monocytes in vitro also saturates and increases with increasing WSS $\tau$ (see [42, Fig. 1). No experimental data exist that quantify the influence of the monocyte concentration in the bloodstream on the amount of adhering monocytes on the endothelium. Here, it is assumed that it is solely dependent on the activation of the endothelium and that there is a surplus of monocytes at all times such that the number of adhering monocytes is independent of the number of monocytes in the flow chamber.

The mathematical submodel is formulated in the same units that are used in the experiments: time $[t]=\mathrm{h}$, concentration of native LDL $[\ell]=\frac{\mu \mathrm{g} \text { protein }}{\mathrm{ml}}$, concentration of modified LDL $[\tilde{\ell}]=\frac{\mu \mathrm{g} \text { protein }}{\mathrm{ml}}$, density of endothelial cells $[e]=\frac{\mathrm{mm}^{2}}{\mathrm{ml}}$, number of adhered monocytes $[m]=\frac{1}{0.1452 \mathrm{~mm}^{2}}$ and WSS exposure $[\tau]=\frac{\text { dyn }}{\mathrm{cm}^{2}}$. The submodel consists of two systems of ODEs for the sequential processes and their associated initial conditions. The first system corresponds to the modification of native LDL by endothelial cells (modification stage)

$$
\begin{gathered}
\frac{\mathrm{d}}{\mathrm{d} t} \ell(t)=-\underbrace{q_{\ell, e} \ell e}_{\begin{array}{c}
\text { modification of native } \\
\text { LDL by endothelial cells }
\end{array}}=-\frac{\mathrm{d}}{\mathrm{d} t} \tilde{\ell}(t), \\
\ell(4 \mathrm{~h})=\ell_{0}, \quad \tilde{\ell}(4 \mathrm{~h})=\tilde{\ell}_{0}, \quad t \in\left[4 \mathrm{~h} ; T_{\mathrm{Mod}}\right]
\end{gathered}
$$


and the second system corresponds to the adhesion and detachment of monocytes (adhesion stage)

$$
\begin{aligned}
\frac{\mathrm{d}}{\mathrm{d} t} m(t) & =P_{m} \underbrace{\left(1-\delta_{m} \frac{k_{m}}{k_{m}+\tilde{\ell}\left(T_{\mathrm{Mod}}\right)}\right)}_{\begin{array}{c}
\text { activation of endothelial cells } \\
\text { by modified LDL }
\end{array}} \underbrace{\left(1-\delta_{\tau} \frac{\tau^{\nu_{\tau}}}{\left(\xi_{\tau}\right)^{\nu_{\tau}}+\tau^{\nu_{\tau}}}\right)}_{\begin{array}{c}
\text { fraction of monocytes not } \\
\text { detached from endothelial } \\
\text { cells by WSS }
\end{array}}, \\
m(0) & =0, \quad t \in\left[0 ; T_{\mathrm{Adh}}\right],
\end{aligned}
$$

where $q_{\ell, e}=2.48 \cdot 10^{-5} \frac{\mathrm{ml}}{\mathrm{hmm}^{2}}$ (see [92], Table 4) and $T_{\mathrm{Mod}}, \ell_{0}, \tilde{\ell}_{0}, e, T_{\mathrm{Adh}}, \tau$ are the experiment-specific parameters, see Table 1. The remaining constants $P_{m}, \delta_{m}, k_{m}, \delta_{\tau}, \xi_{\tau}$ and $\nu_{\tau}$ are the unknown parameters of the submodel. They are estimated by least-squares fitting of the simulated number of adhered monocytes $m\left(T_{\mathrm{Adh}}\right)$ to the experimental results in [42].

\subsubsection{Macrophage Recruitment}

The experiments by Jeng et al. 42] considered only the adhesion of monocytes in the absence of flow and their successive detachment under various flow conditions. When monocytes migrate from the bloodstream into the artery wall in vivo, however, the attachment of monocytes takes place under pulsatile flow conditions. No experimental data exist that allow us to quantify the adhesion of monocytes on activated endothelial cells under such flow conditions. The submodel of macrophage recruitment is based on the previous submodel of monocyte adhesion and the experimental results by Alon et al. [2].

Mathematical submodel The influence of the WSS on the adhesion of Tlymphocytes on VCAM-1 saturated plastic slides was investigated by Alon et al. [2]. The experimental results show that the adhesion of cells under flow conditions vanishes when they are exposed to high WSS (see 2, Fig. 1). In this submodel, the vanishing adhesion is accounted for by neglecting the limiting of the saturation kinetic with respect to WSS $\tau$, i.e., by using $\delta_{\tau}=1$ for the in vivo setup. Due to the presence of MCP-1 and other monocyte-attracting molecules in vivo, it is assumed that each monocyte that adheres and remains bound under heavy flow conditions also migrates into the intima. Further, the time scale of atherosclerosis progression is sufficiently large that the relatively fast differentiation of monocytes into macrophages [61 can be approximated by an instantaneous differentiation. The complete submodel for the rate of recruitment of macrophages $r_{m}$ is therefore:

$$
r_{m}(\tilde{\ell}, \tau)=P_{m}\left(1-\delta_{m} \frac{k_{m}}{k_{m}+\tilde{\ell}}\right) \frac{\left(\xi_{\tau}\right)^{\nu_{\tau}}}{\left(\xi_{\tau}\right)^{\nu_{\tau}}+\tau^{\nu_{\tau}}},
$$

where all parameters have been introduced previously. 


\subsubsection{Lipoprotein Fluxes}

Lipoproteins, such as LDL and HDL are transported through the endothelium at rates dependent on their concentrations in blood and on the local blood flow conditions. The endothelium is frequently modeled as a semi-permeable membrane described by the two equations of Kedem and Katchalsky 45 103, 10, 43, 74, 93. To this end, blood plasma is treated as solvent, whereas lipoproteins are interpreted as blood solutes. The first Kedem-Katchalsky equation estimates the total volume flux of the solvent as the difference of fluid and osmotic pressure gradient driven fluxes. The second equation of Kedem and Katchalsky describes the flux of the solute as sum of a concentration gradientdriven diffusive flux and an advective flux due to the transport with solvent's flow. The applicability of the Kedem-Katchalsky equations to trans-endothelial transport was investigated by Thomas and Mikulecky [90.

Mathematical submodel The transport of native LDL $\ell$ in the blood plasma through the endothelium is governed by the second Kedem-Katchalsky equation [45. Thus, the total solute flux of native LDL $r_{\ell}$ into the intima can be subdivided into a diffusive and an advective flux with the blood plasma

$r_{\ell}(\ell, p,\|\boldsymbol{\tau}\|)=\underbrace{P_{\ell} s_{\mathrm{P}}(\|\boldsymbol{\tau}\|)\left(\eta_{\ell}-\ell\right)}_{\text {diffusive flux }}+\underbrace{\left(1-\sigma_{\mathrm{F}, \ell}\right)\left(\omega_{\ell} \eta_{\ell}+\left(1-\omega_{\ell}\right) \ell\right) J_{\mathrm{Vol}}(p,\|\boldsymbol{\tau}\|)}_{\text {advective flux } r_{\mathrm{Adv}, \ell}(\ell, p,\|\boldsymbol{\tau}\|)}$,

where $P_{\ell}$ is the diffusive permeability of LDL, $\eta_{\ell}$ is the concentration of native LDL in blood, $\sigma_{\mathrm{F}, \ell}$ is the filtration reflection coefficient with respect to LDL and $\omega_{\ell}$ is the weighting factor for the average LDL concentration within the endothelium layer. The volume flux of blood plasma through the endothelium $J_{\text {Vol }}$ is given by the first Kedem-Katchalsky equation without osmotic flux 89, 40

$$
J_{\mathrm{Vol}}(p,\|\boldsymbol{\tau}\|)=L_{p} s_{\mathrm{L}}(\|\boldsymbol{\tau}\|)\left(\eta_{p}-p\right)
$$

where $\eta_{p}$ and $p$ correspond to the luminal blood pressure and the subendothelial blood plasma pressure, respectively. The diffusive permeability and hydraulic conductivity depend on the 2-norm of the local WSS $\|\boldsymbol{\tau}\|$ that the model plaque is exposed to. Here, $\tau$ corresponds to the WSS vector time-averaged over the pulsatile cardiac cycle, cf. [93,72,6,53,48, The monotonically decreasing (diffusive) permeability scaling factor (PSF) from 93 is employed for the function $s_{\mathrm{P}}$ for the dependence of diffusive permeability on WSS:

$$
s_{\mathrm{P}}(\|\boldsymbol{\tau}\|)=\frac{1}{\ln (2)} \ln \left(1+\zeta_{\tau} \frac{\gamma_{\tau}}{\|\boldsymbol{\tau}\|+\gamma_{\tau}}\right) .
$$

In contrast to the diffusive permeability, there is direct experimental evidence that the hydraulic conductivity increases as endothelial cells are exposed to 
increased WSS $\|\boldsymbol{\tau}\|$ [81,13. Therefore, the hydraulic conductivity is altered by the following monotonically increasing function from literature [76, 85]

$$
s_{\mathrm{L}}(\|\boldsymbol{\tau}\|)=\gamma_{p} \ln \left(\mu_{p}\|\boldsymbol{\tau}\|+\xi_{p}\right),
$$

which is subsequently called (hydraulic) conductivity scaling factor (CSF).

Due to their related structure and size, the transport mechanisms of modified LDL and native HDL here are assumed to be the same as that of native LDL. Hence, the fluxes of modified LDL $r_{\ell}$ and HDL $r_{h}$ are also modeled by second Kedem-Katchalsky equation:

$$
\begin{aligned}
r_{\tilde{\ell}}(\tilde{\ell}, p,\|\boldsymbol{\tau}\|)=P_{\tilde{\ell}} s_{\mathrm{P}}(\|\boldsymbol{\tau}\|) & \left(\eta_{\tilde{\ell}}-\tilde{\ell}\right) \\
& +\left(1-\sigma_{\mathrm{F}, \tilde{\ell}}\right)\left(\omega_{\tilde{\ell}} \eta_{\tilde{\ell}}+\left(1-\omega_{\tilde{\ell}}\right) \tilde{\ell}\right) J_{\mathrm{Vol}}(p,\|\boldsymbol{\tau}\|), \\
r_{h}(h, p,\|\boldsymbol{\tau}\|)=P_{h} s_{\mathrm{P}}(\|\boldsymbol{\tau}\|) & \left(\eta_{h}-h\right) \\
& +\left(1-\sigma_{\mathrm{F}, h}\right)\left(\omega_{h} \eta_{h}+\left(1-\omega_{h}\right) h\right) J_{\mathrm{Vol}}(p,\|\boldsymbol{\tau}\|) .
\end{aligned}
$$

\subsection{Spatially Resolved Model of Early Atherosclerosis}

The penetration and transport of lipoproteins and cells as well as the immunological and inflammatory processes of atherosclerosis take place in the intima and adjacent media. Any spatial model of these species must therefore consider both layers and their main transport barriers - the endothelium and the internal elastic lamina which separates intima and media. However, monocytes actively transmigrate from the intima into the media [60,80] and the transport rates of lipoproteins through the internal elastic lamina are more than two orders of magnitude faster compared to those through the endothelium 44 [103]. As a consequence, the internal elastic lamina represents solely a minor transport barrier for LDL, HDL and macrophages. We therefore neglect the internal elastic lamina and use a fluid-wall model [74], where the endothelium is the only transport barrier in the model. Further, the immunological and inflammatory processes and species transport in atherosclerosis take place on a time scale of days or weeks, where the deformation of the intima and media due to the pulsatile blood flow which occurs on a time scale of seconds can be ignored [93].

In the spatially resolved model, all species are modeled by temporal and spatially continuous concentrations with the unit of number per volume. We denote the intima-media domain by $\Omega$, the inlet boundary by $\Gamma_{\text {In }}$, the outlet boundary by $\Gamma_{\text {Out }}$, the endothelial boundary by $\Gamma_{\text {End }}$ and the media-adventitia boundary by $\Gamma_{\mathrm{Adv}}$. For a schematic overview of the domains and boundaries of the spatially resolved model of early atherosclerosis, see Figure 2

\subsubsection{Transmural Flow}

As frequently done in the literature [47,44, 94, 10, 74, 70, we model the transmural filtration flow within the artery wall as a purely pressure gradient-driven 


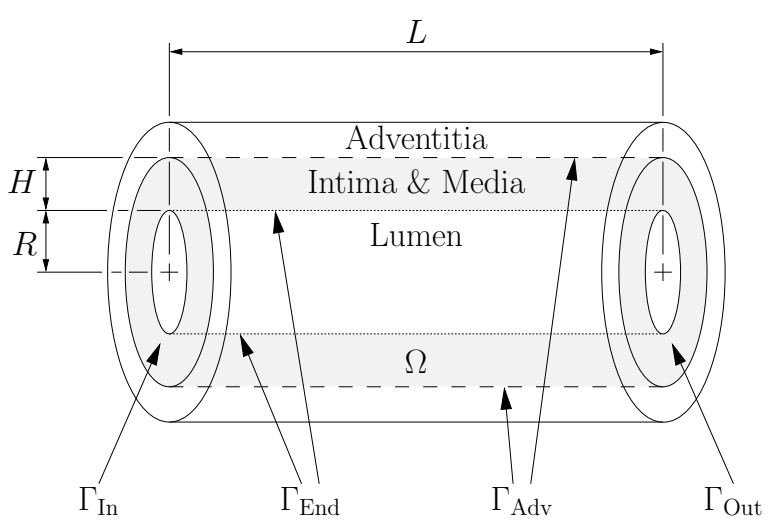

Fig. 2 Schematic overview of the domain (gray) and boundaries of the spatially resolved model of early atherosclerosis: intima-media domain $\Omega$, inlet boundary $\Gamma_{\mathrm{In}}$, endothelial boundary $\Gamma_{\text {End }}$, media-adventitia boundary $\Gamma_{\mathrm{Adv}}$ and outlet boundary $\Gamma_{\text {Out }}$. The domain is characterized by the lumen radius $R$, intima-media thickness $H$ and segment length $L$.

incompressible flow. Since the intima-media domain $\Omega$ does not deform it will have a constant porosity $\phi$. The transmural flow $\boldsymbol{u}$ is thus described by Darcy's law and the incompressibility equation [3, 18, for all $t \geq 0$ and $\boldsymbol{x} \in \Omega$ :

$$
\begin{aligned}
\boldsymbol{u}(t, \boldsymbol{x}) & =-\frac{K}{\phi \mu} \nabla p(t, \boldsymbol{x}), \\
\nabla \cdot \boldsymbol{u}(t, \boldsymbol{x}) & =0
\end{aligned}
$$

where $K$ and $\mu$ are the Darcy permeability and dynamic viscosity, respectively. To simplify the notation we subsequently drop the temporal and spatial arguments whenever they are not crucial for understanding. Together, both equations yield the Poisson equation for the intimal and medial blood plasma pressure $p$ on the intima-media domain $\Omega$ :

$$
-\nabla \cdot\left(\frac{K}{\phi \mu} \nabla p\right)=0
$$

On the media-adventitia boundary $\Gamma_{\mathrm{Adv}}$ a constant pressure corresponding to the adventitial pressure $p_{\mathrm{Adv}}$ is prescribed as a Dirichlet boundary condition:

$$
p=p_{\mathrm{Adv}} \text {. }
$$

The volume flux $J_{\mathrm{Vol}}$ through the endothelial boundary $\Gamma_{\text {End }}$ is described by the first Kedem-Katchalsky equation:

$$
-\frac{K}{\phi \mu} \nabla p \cdot \boldsymbol{n}_{\mathrm{End}} \stackrel{9}{=} \boldsymbol{u} \cdot \boldsymbol{n}_{\mathrm{End}}=J_{\mathrm{Vol}}(p,\|\boldsymbol{\tau}\|) \stackrel{5}{=} L_{p} s_{\mathrm{L}}(\|\boldsymbol{\tau}\|)\left(\eta_{p}-p\right) .
$$


Here, $\boldsymbol{n}_{\star}$ denotes the outward normal to surface $\Gamma_{\star}$, cf. Figure 2 On the inlet and outlet boundaries $\Gamma_{\text {In }}$ and $\Gamma_{\text {Out }}$ no-flux conditions are applied:

$$
\begin{aligned}
-\frac{K}{\phi \mu} \nabla p \cdot \boldsymbol{n}_{\text {In }} & =0, \\
-\frac{K}{\phi \mu} \nabla p \cdot \boldsymbol{n}_{\text {Out }} & =0 .
\end{aligned}
$$

\subsubsection{Interaction and Transport of Species}

The model for the interaction and transport of lipoproteins and macrophages is an extension and generalization of the non-spatial model given in [92] to a spatially resolved model that includes diffusive and advective transport and appropriate boundary conditions. The general framework of the spatially resolved model is given by the porous medium-advection-diffusion-reaction equation, see $[29,3,18]$. The submodels for the concentrations of native LDL $\ell$, modified LDL $\tilde{\ell}$ and HDL $h$ on the intima-media domain $\Omega$ are

$$
\begin{aligned}
& \frac{\partial}{\partial t} \ell(t, \boldsymbol{x})+\underbrace{\nabla \cdot\left(K_{\ell} \ell \boldsymbol{u}\right)}_{\begin{array}{c}
\text { advection of } \\
\text { native LDL with } \\
\text { transmural flow }
\end{array}}-\underbrace{\nabla \cdot\left(D_{\mathrm{Eff}, \ell} \nabla \ell\right)}_{\begin{array}{c}
\text { diffusion of } \\
\text { native LDL }
\end{array}}=-\underbrace{\mu_{\ell} \frac{\ell^{n_{\ell}}}{\left(\xi_{\ell}\right)^{n_{\ell}}+\ell^{n_{\ell}}} m}_{\begin{array}{c}
\text { ingestion of native } \\
\text { LDL by macrophages }
\end{array}} \\
& -\underbrace{q_{\ell, m} \ell m \frac{\left(k_{h}\right)^{n_{h}}}{\left(k_{h}\right)^{n_{h}}+h^{n_{h}}}}, \\
& \text { modification of native } \\
& \begin{array}{l}
\text { LDL by macrophages } \\
\text { and inhibition by HDL }
\end{array} \\
& \frac{\partial}{\partial t} \tilde{\ell}(t, \boldsymbol{x})+\underbrace{\nabla \cdot\left(K_{\tilde{\ell}} \tilde{\ell} \boldsymbol{u}\right)}_{\begin{array}{c}
\text { advection of } \\
\text { modified LDL with } \\
\text { transmural flow }
\end{array}}-\underbrace{\nabla \cdot\left(D_{\mathrm{Eff}, \tilde{\ell}} \nabla \tilde{\ell}\right)}_{\begin{array}{c}
\text { diffusion of } \\
\text { modified LDL }
\end{array}}=-\underbrace{\mu_{\tilde{\ell}} \frac{\tilde{\ell}^{n_{\tilde{\ell}}}}{\left(\xi_{\tilde{\ell}}\right)^{n_{\tilde{\ell}}}+\tilde{\ell}^{n_{\tilde{\ell}}}} m}_{\begin{array}{c}
\text { ingestion of modified } \\
\text { LDL by macrophages }
\end{array}} \\
& +\underbrace{q_{\ell, m} \ell m \frac{\left(k_{h}\right)^{n_{h}}}{\left(k_{h}\right)^{n_{h}}+h^{n_{h}}}}, \\
& \text { modification of native } \\
& \text { and inhibition by HDL } \\
& \frac{\partial}{\partial t} h(t, \boldsymbol{x})+\underbrace{\boldsymbol{\nabla} \cdot\left(K_{h} h \boldsymbol{u}\right)}_{\begin{array}{c}
\text { advection of } \\
\text { HDL with } \\
\text { transmural flow }
\end{array}}-\underbrace{\boldsymbol{\nabla} \cdot\left(D_{\mathrm{Eff}, h} \nabla h\right)}_{\text {diffusion of HDL }}=-\underbrace{q_{h, m} h m}_{\begin{array}{c}
\text { modification of HDL } \\
\text { by macrophages }
\end{array}},
\end{aligned}
$$

where the advection of lipoproteins due to the transmural filtration flow is governed by the gradient of the blood plasma pressure $p$, see Eq. (9). The reduction of the advective transport due to interactions with the artery wall is taken into account by the hindrance coefficients $K_{\ell}, K_{\tilde{\ell}}$ and $K_{h}$ [29,70].

Macrophages and their intracellular cholesterols are not transported by advection with the transmural flow because they are several orders of magnitude 
larger than lipoprotein particles [62. Thus, the models for the total concentration of intracellular free cholesterol $f$, the total concentration of intracellular cholesterol ester $b$ and the density of macrophages $m$ on the intima-media domain $\Omega$ are

$$
\begin{aligned}
& \frac{\partial}{\partial t} f(t, \boldsymbol{x})-\underbrace{\nabla \cdot\left(D_{\mathrm{Eff}, f} \nabla f\right)}_{\begin{array}{c}
\text { diffusion of } \\
\text { free cholesterol }
\end{array}}=+N_{f} \underbrace{\mu_{\ell} \frac{\ell^{n_{\ell}}}{\left(\xi_{\ell}\right)^{n_{\ell}}+\ell^{n_{\ell}}} m}_{\begin{array}{c}
\text { ingestion of native } \\
\text { LDL by macrophages }
\end{array}}+N_{f} \underbrace{\mu_{\tilde{\ell}} \frac{(\tilde{\ell})^{n_{\tilde{\ell}}}}{\left(\xi_{\tilde{\ell}}\right)^{n_{\tilde{\ell}}}+(\tilde{\ell})^{n_{\tilde{\ell}}}} m}_{\begin{array}{c}
\text { ingestion of modified } \\
\text { LDL by macrophages }
\end{array}} \\
& -\underbrace{k_{f} \frac{\left(f-f_{\mathrm{Min}} m\right)^{2}}{f_{\mathrm{Max}} m-f}}_{\begin{array}{c}
\text { esterification of } \\
\text { free cholesterol }
\end{array}}+\underbrace{k_{b} b}_{\begin{array}{c}
\text { hydrolysis of } \\
\text { cholesterol ester }
\end{array}} \\
& \text { - } \underbrace{\mu_{f} \frac{h^{n_{f}}}{\left(\xi_{f}\right)^{n_{f}}+h^{n_{f}}}\left(f-f_{\mathrm{Min}} m\right)}_{\text {cholesterol efflux from macrophages to HDL }}, \\
& \frac{\partial}{\partial t} b(t, \boldsymbol{x})-\underbrace{\boldsymbol{\nabla} \cdot\left(D_{\mathrm{Eff}, b} \nabla b\right)}_{\begin{array}{c}
\text { diffusion of } \\
\text { cholesterol ester }
\end{array}}=+\underbrace{k_{f} \frac{\left(f-f_{\mathrm{Min}} m\right)^{2}}{f_{\mathrm{Max}} m-f}}_{\begin{array}{c}
\text { esterification of } \\
\text { free cholesterol }
\end{array}}-\underbrace{k_{b} b}_{\begin{array}{c}
\text { hydrolysis of } \\
\text { cholesterol ester }
\end{array}}, \\
& \frac{\partial}{\partial t} m(t, \boldsymbol{x})-\underbrace{\boldsymbol{\nabla} \cdot\left(D_{\mathrm{Eff}, m} \nabla m\right)}_{\begin{array}{c}
\text { diffusion of } \\
\text { macrophages }
\end{array}}=-\underbrace{\mu_{m} \frac{f^{n_{m}}}{\left(\xi_{m} m\right)^{n_{m}}+f^{n_{m}}} m}_{\text {apoptosis of macrophages }} .
\end{aligned}
$$

On the inlet, outlet and media-adventitia boundaries $\Gamma_{\mathrm{In}}, \Gamma_{\text {Out }}$ and $\Gamma_{\mathrm{Adv}}$, no-flux conditions are applied. In accordance with [92], the influx boundary 
conditions on the endothelial boundary $\Gamma_{\text {End }}$ are given by

$$
\begin{aligned}
& -\left(K_{\ell} \ell \boldsymbol{u}-D_{\mathrm{Eff}, \ell} \nabla \ell\right) \cdot \boldsymbol{n}_{\mathrm{End}}=-\underbrace{q_{\ell, e} \ell \frac{\left(k_{h}\right)^{n_{h}}}{\left(k_{h}\right)^{n_{h}}+h^{n_{h}}}}_{\begin{array}{c}
\text { modification of native } \\
\text { LDL by endothelial cells } \\
\text { and inhibition by HDL }
\end{array}}+\underbrace{r_{\ell}(\ell, p,\|\boldsymbol{\tau}\|)}_{\begin{array}{c}
\text { influx of native LDL } \\
\text { from lumen }
\end{array}}, \\
& -\left(K_{\tilde{\ell}} \tilde{\ell} \boldsymbol{u}-D_{\mathrm{Eff}, \tilde{\ell}} \nabla \tilde{\ell}\right) \cdot \boldsymbol{n}_{\mathrm{End}}=+\underbrace{q_{\ell, e} \ell \frac{\left(k_{h}\right)^{n_{h}}}{\left(k_{h}\right)^{n_{h}}+h^{n_{h}}}}_{\begin{array}{c}
\text { modification of native } \\
\text { LDL by endothelial cells } \\
\text { and inhibition by HDL }
\end{array}}+\underbrace{\left.r_{\tilde{\ell}} \tilde{\ell}, p,\|\boldsymbol{\tau}\|\right)}_{\begin{array}{c}
\text { ffflux of modified LDL } \\
\text { to lumen }
\end{array}}, \\
& -\left(K_{h} h \boldsymbol{u}-D_{\mathrm{Eff}, h} \nabla h\right) \cdot \boldsymbol{n}_{\mathrm{End}}=-\underbrace{q_{h, e} h}_{\begin{array}{c}
\text { modification of HDL } \\
\text { by endothelial cells }
\end{array}}+\underbrace{r_{h}(h, p,\|\boldsymbol{\tau}\|)}_{\begin{array}{c}
\text { influx of native HDL } \\
\text { from lumen }
\end{array}}, \\
& -\left(-D_{\mathrm{Eff}, f} \nabla f\right) \cdot \boldsymbol{n}_{\mathrm{End}}=+f_{\mathrm{In}} \underbrace{r_{m}(\tilde{\ell},\|\boldsymbol{\tau}\|)}_{\begin{array}{c}
\text { recruitment of } \\
\text { macrophages }
\end{array}}, \\
& -\left(-D_{\mathrm{Eff}, b} \nabla b\right) \cdot \boldsymbol{n}_{\mathrm{End}}=0, \\
& -\left(-D_{\mathrm{Eff}, m} \nabla m\right) \cdot \boldsymbol{n}_{\mathrm{End}}=+\underbrace{r_{m}(\tilde{\ell},\|\boldsymbol{\tau}\|)}_{\begin{array}{c}
\text { recruitment of } \\
\text { macrophages }
\end{array}},
\end{aligned}
$$

where the functions $r_{\ell}, r_{\tilde{\ell}}, r_{h}$ and $r_{m}$ are given by Eqs. (3), (4) and (8).

\subsubsection{Initial Conditions}

A spatially constant initial blood plasma pressure $p_{0}$ is utilized

$$
p(0, \boldsymbol{x})=p_{0},
$$

and spatially constant initial concentrations analogous to the ODE model in 92. are employed

$$
\begin{aligned}
\ell(0, \boldsymbol{x}) & =0, \quad \tilde{\ell}(0, \boldsymbol{x})=\tilde{\ell}_{0}, \quad h(0, \boldsymbol{x})=0, \\
f(0, \boldsymbol{x}) & =f_{0} m_{0}, \quad b(0, \boldsymbol{x})=b_{0} m_{0}, \quad m(0, \boldsymbol{x})=m_{0} .
\end{aligned}
$$

The initial amount of intracellular free cholesterol per macrophage $f_{0}$, the initial amount of cholesterol ester per macrophage $b_{0}$, and the initial density of macrophages $m_{0}$ are defined as in [92]:

$$
f_{0}=1.5 f_{\mathrm{In}}, \quad m_{0}=\frac{r_{m}\left(\tilde{\ell}_{0},\|\boldsymbol{\tau}\|\right)}{H} \frac{\left(\xi_{m}\right)^{n_{m}}+\left(f_{0}\right)^{n_{m}}}{\mu_{m}\left(f_{0}\right)^{n_{m}}}, \quad b_{0}=\frac{k_{f}}{k_{b}} \frac{\left(f_{0}-f_{\mathrm{Min}}\right)^{2}}{f_{\mathrm{Max}}-f_{0}} .
$$

Remark 1 The parameters and terms on the right-hand sides of Eqs. (15), (16), (18) and 190 match the notations and definitions introduced in a previous work 92 . Therein, detailed derivations and underlaying assumptions are studied and discussed. 


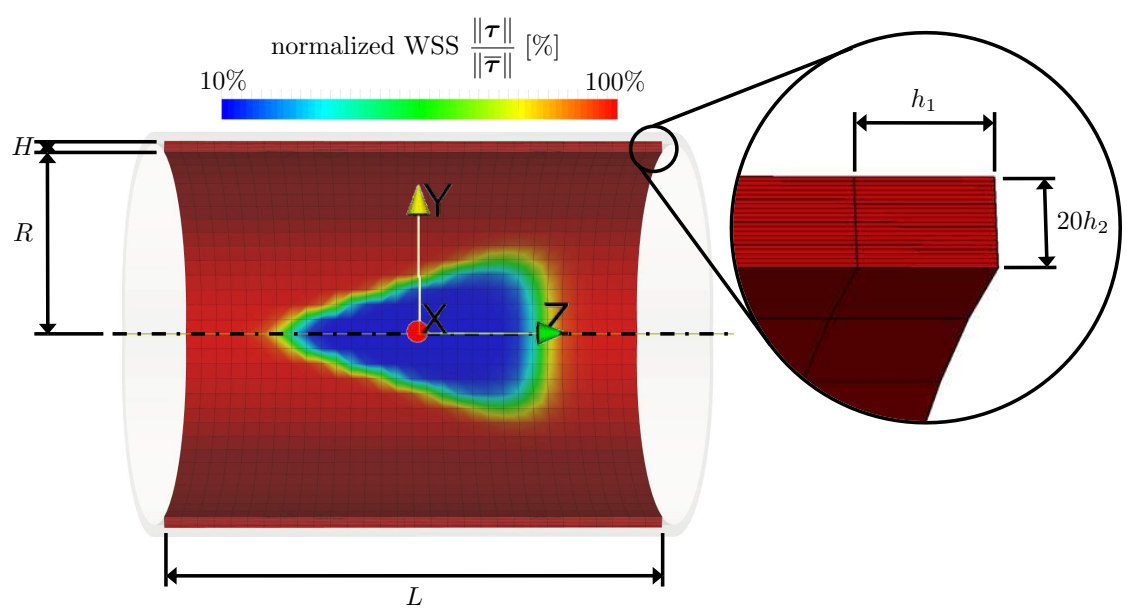

Fig. 3 Finite element mesh for the spatially resolved model with $h_{1}=6.0$. $10^{-2} \mathrm{~mm}$ and $h_{2}=2.0 \cdot 10^{-3} \mathrm{~mm}$ and synthetic inhomogeneous normalized WSS pattern $\frac{\|\boldsymbol{\tau}\|}{\|\overline{\boldsymbol{\tau}}\|}$ used in calculations.

\section{Results}

In this section we present the models' parameters and computational results. All numerical computations for ODE models were performed using MATLAB (The MathWorks Inc., Natick, Massachusetts, USA, 2000). The solution of the spatially resolved model was done using our in-house finite element code. The spatially resolved model is discretized in time and space by the one-step- $\theta$ scheme (with $\theta=0.6$ ) and the finite element method [22], respectively. An overview of the finite element mesh for the idealized intima-media domain $\Omega$ discretized with bilinear hexahedral elements is given in Figure 3.

\subsection{Model Parameters}

For the spatially resolved model, we consider a simplified geometrical setup of the murine physiology of the aortic arch. To this end, the dimensions corresponding to the example in $[93$ are employed: lumen radius $R=0.57 \mathrm{~mm}$, intima-media thickness $H=0.04 \mathrm{~mm}(\approx 50 \%$ of total wall thickness [28, 38 , 44]), and segment length $L=3 R \approx 1.8 \mathrm{~mm}$, cf. Figure 3 .

In Appendix A, a complete parameter set for the spatially resolved model is derived from least-squares fits to experimental results and existing literature. An overview of estimated parameters is given in Table 2.

It is common practice to assume a reference WSS value $\|\overline{\boldsymbol{\tau}}\|$ to assess the endothelial permeability with respect to lipoproteins [93, 85, 10]. Even though the distribution of the reference WSS value within an animal is more or less uniform [79], it varies significantly in between different animal models [14]. 
Table 2 Fitted and estimated parameters of the spatially resolved model. Parameters are given in the units $\mathrm{mm}, \mathrm{g}, \mathrm{h}$ and $\mathrm{Pa}$. The remaining parameters of the model are given in 92 , Table 4.

\begin{tabular}{|c|c|c|c|}
\hline Parameter & Description & Value & $\begin{array}{l}\text { Fitted to } / \\
\text { source }\end{array}$ \\
\hline$R$ & Lumen radius & $5.7 \cdot 10^{-1} \mathrm{~mm}$ & - $93 \mid$ \\
\hline$H$ & Intima-media thickness & $4.00 \cdot 10^{-2} \mathrm{~mm}$ & 93.28 .38 .44 \\
\hline$L$ & Segment length & $1.8 \mathrm{~mm}$ & $\approx 3 R$ \\
\hline$P_{m}$ & Rate of recruitment of $m$ & $3.05 \cdot 10^{3} \frac{1}{\mathrm{hmm^{2 }}}$ & 42 \\
\hline$\delta_{m}$ & Fraction of saturation of recruitment of $m$ by $\tilde{\ell}$ & $68.62 \%$ & 42 \\
\hline$k_{m}$ & Saturation of recruitment of $m$ by $\tilde{\ell}$ & $7.38 \cdot 10^{6} \frac{1}{\mathrm{~mm}^{3}}$ & 42 \\
\hline$\xi_{\tau}$ & Saturation of recruitment of $m$ by $\tau$ & Table $3{ }^{3}$ & 42 \\
\hline$\nu_{\tau}$ & Exponent of recruitment of $m$ by $\tau$ & $1.18^{\square}$ & 42 \\
\hline$D_{\mathrm{Eff}, m}$ & Effective diffusion coefficient of $m$ & $3.6 \cdot 10^{-6} \frac{\mathrm{mm}^{2}}{\mathrm{~h}_{0}}$ & 15.30 \\
\hline$D_{\mathrm{Eff}, f}$ & Effective diffusion coefficient of $f$ & $3.6 \cdot 10^{-6} \frac{\mathrm{mm}^{2}}{\mathrm{~h}}$ & $=D_{\mathrm{Eff}, m}$ \\
\hline$D_{\mathrm{Eff}, b}$ & Effective diffusion coefficient of $b$ & $3.6 \cdot 10^{-6} \frac{\mathrm{mm}^{2}}{\mathrm{~h}}$ & $=D_{\mathrm{Eff}, m}$ \\
\hline$P_{\ell}$ & Diffusive permeability of $\ell$ & $6.12 \cdot 10^{-5} \frac{\mathrm{n} m}{\mathrm{~h}}$ & [95] \\
\hline$\zeta_{\tau}$ & Permeability scaling factor parameter & $31{ }^{11}$ & 93 \\
\hline$\gamma_{\tau}$ & Permeability scaling factor parameter & Table 3 & 93 \\
\hline$\sigma_{\mathrm{F}, \ell}$ & Filtration reflection coefficient of $\ell$ & $9.97 \cdot 10^{-1}$ & $44,103.86$ \\
\hline$\omega_{\ell}$ & Weighting factor of $\ell$ & $9.99 \cdot 10^{-1}$ & Eq. 23], 29] \\
\hline$L_{p}$ & Hydraulic conductivity & $4.26 \cdot 10^{-5} \frac{\mathrm{mm}}{\mathrm{Pah}}$ & 891 \\
\hline$\gamma_{p}$ & Conductivity scaling factor parameter & $1.31 \cdot 10^{-1^{\mathrm{an}}}$ & 81.85 \\
\hline$\mu_{p}$ & Conductivity scaling factor parameter & Table 3 & 81.85 \\
\hline$\xi_{p}$ & Conductivity scaling factor parameter & $1.86 \cdot 10^{1}$ & 81.85 \\
\hline $\begin{array}{c}D_{\mathrm{Eff}, \ell} \\
K_{\ell}\end{array}$ & $\begin{array}{c}\text { Effective diffusion coefficient of } \ell \\
\text { Hindrance coefficient of } \ell\end{array}$ & $\begin{array}{r}1.26 \cdot 10^{-2} \frac{\mathrm{mm}^{2}}{\mathrm{~h}} \\
1.17 \cdot 10^{-1^{3}}\end{array}$ & $\frac{94,86,10 \mid}{29,70 \mid}$ \\
\hline$P_{\tilde{\ell}}$ & Diffusive permeability of $\tilde{\ell}$ & $6.12 \cdot 10^{-5} \frac{\mathrm{mm}}{\mathrm{h}}$ & $=P_{\ell}$ \\
\hline$\eta_{\tilde{\ell}}^{\ell}$ & Concentration of $\tilde{\ell}$ in blood & $0.0{\frac{1}{m^{3}}}^{h}$ & 23 \\
\hline$\sigma_{\mathrm{F}, \tilde{\ell}}$ & Filtration reflection coefficient of $\tilde{\ell}$ & $9.97 \cdot 10^{-1}$ & $=\sigma_{\mathrm{F}, \ell}$ \\
\hline$\omega_{\tilde{\ell}}$ & Weighting factor of $\tilde{\ell}$ & $9.99 \cdot 10^{-1}$ & $=\omega_{\ell}$ \\
\hline $\begin{array}{c}D_{\mathrm{Eff}, \tilde{\ell}} \\
K_{\tilde{\imath}}\end{array}$ & $\begin{array}{c}\text { Effective diffusion coefficient of } \tilde{\ell} \\
\text { Hindrance coefficient of } \tilde{\ell}\end{array}$ & $\begin{array}{c}1.26 \cdot 10^{-2} \frac{\mathrm{mm}^{2}}{\mathrm{~h}} \\
1.17 \cdot 10^{-1}\end{array}$ & $\begin{array}{l}=D_{\mathrm{Eff}, \ell} \\
=K\end{array}$ \\
\hline$P_{h}$ & Diffusive permeability of $h$ & $1.14 \cdot 10^{-4} \frac{\mathrm{mm}}{\mathrm{h}}$ & $\left.=1.87 P_{\ell}, 83\right]$ \\
\hline$\sigma_{\mathrm{F}, h}$ & Filtration reflection coefficient of $h$ & $9.18 \cdot 10^{-1^{\mathrm{h}}}$ & Eq. 25, 441 \\
\hline$\omega_{h}$ & Weighting factor of $h$ & $9.98 \cdot 10^{-1}$ & Eq. 23, 29] \\
\hline$q_{h, m}$ & Rate of modification of $h$ by $m$ & $1.64 \cdot 10^{-4} \frac{\mathrm{mm}^{3}}{\mathrm{~h}}$ & $=\frac{q \ell, m}{7.6} \frac{N_{\tilde{\ell}}}{N_{\tilde{h}}}$ \\
\hline$q_{h, e}$ & Rate of modification of $h$ by $e$ & $6.21 \cdot 10^{-2} \frac{\mathrm{mm}}{\mathrm{h}}$ & $=\frac{q_{\ell, e}}{7.6} \frac{N_{\tilde{\ell}}^{n}}{N_{\tilde{h}}}$ \\
\hline$D_{\mathrm{Eff}, h}$ & Effective diffusion coefficient of $h$ & $2.94 \cdot 10^{-2} \frac{\mathrm{mm}^{2}}{\mathrm{~h}}$ & Eq. 27, 44 \\
\hline$\frac{K_{h}}{\phi}$ & $\begin{array}{c}\text { Hindrance coefficient of } h \\
\text { Porosity of media }\end{array}$ & $6.27 \cdot 10^{-1}$ & $\frac{\text { Eq. }[26],[29]}{\mid 1.74]}$ \\
\hline K & $\begin{array}{l}\text { Porosity of media } \\
\text { Darcy permeability of artery wall }\end{array}$ & $1.11 \cdot 10^{-14} \mathrm{~mm}^{2}$ & Eq. $\sqrt{29}$ \\
\hline$\varrho$ & Mass density of blood plasma & $1.06 \cdot 10^{-3} \frac{\mathrm{g}}{\mathrm{mm}^{3}}$ & 103 \\
\hline$\mu$ & Dynamic viscosity of blood plasma & $2.0 \cdot 10^{-7} \mathrm{Pah}$ & 74.103 \\
\hline$\eta_{p}$ & Luminal blood pressure & $1.33 \cdot 10^{4} \mathrm{~Pa}$ & 1,69.98] \\
\hline$p_{\mathrm{Adv}}$ & Adventitial blood plasma pressure & $4.0 \cdot 10^{3} \mathrm{~Pa}$ & 1.69 .103 \\
\hline
\end{tabular}

Abbreviations: low-density lipoproteins, $\ell$; modified low-density lipoproteins, $\tilde{\ell}$; high-density lipoproteins, $h$; endothelial cells, $e$; macrophages, $m$; intracellular free cholesterol, $f$; intracellular cholesterol ester, $b$; wall shear stresses, $\tau$. 
Therefore, the parameters of the PSF and CSF must be adapted to match the physiology of the animal under consideration, i.e., murine physiology. A tabular overview for different animal models and the normalized case is given in Table 3. In the following, we always use normalized WSS $\frac{\|\boldsymbol{\tau}\|}{\|\overline{\boldsymbol{\tau}}\|}$ values, as is frequently done in the literature [44, 10, 15, 85].

Table 3 Wall shear stress-dependent parameters for different animal models and the normalized case.

\begin{tabular}{|l||c|c|c|c|}
\hline Species Quantity & $\|\overline{\boldsymbol{\tau}}\|[\mathrm{Pa}]$ & $\xi_{\tau}[\mathrm{Pa}]$ & $\gamma_{\tau}[\mathrm{Pa}]$ & $\mu_{p}\left[\frac{1}{\mathrm{~Pa}}\right]$ \\
\hline \hline Normalized & 1 & $1.68 \cdot 10^{-1}$ & $3.33 \cdot 10^{-2}$ & $20.88 \cdot 10^{2}$ \\
Human & 1.16 & $1.95 \cdot 10^{-1}$ & $3.87 \cdot 10^{-2}$ & $18.00 \cdot 10^{2}$ \\
Dog & 1.61 & $2.71 \cdot 10^{-1}$ & $5.37 \cdot 10^{-2}$ & $12.97 \cdot 10^{2}$ \\
Rabbit & 2.38 & $4.00 \cdot 10^{-1}$ & $7.93 \cdot 10^{-2}$ & $8.77 \cdot 10^{2}$ \\
Rat & 4.71 & $7.92 \cdot 10^{-1}$ & $15.70 \cdot 10^{-2}$ & $4.43 \cdot 10^{2}$ \\
Mouse & 6.52 & $10.96 \cdot 10^{-1}$ & $21.73 \cdot 10^{-2}$ & $3.20 \cdot 10^{2}$ \\
\hline Fitted to / source & {$[14$} & {$[\underline{42}$} & {$[\underline{93}$} & {$[8185$} \\
\hline
\end{tabular}

It remains to specify the concentrations of native LDL $\eta_{\ell}$ and HDL $\eta_{h}$ in blood and the WSS $\|\boldsymbol{\tau}\|$ that the model plaque is exposed to in vivo. These parameters characterize the physiology and diet of the plaque's host and the position of the plaque in the cardiovascular system. Thus, they cannot be set at fixed values, but their full ranges must be considered. An overview of the full spectrum of LDL and HDL blood cholesterol concentrations as well as WSS is given in Table 4. The ranges of interest, however, lie in the lower ends of the WSS-spectrum as these are associated with the development of atherosclerotic plaques in humans 68. If not specified otherwise, we use a concentration of LDL cholesterol $\eta_{\ell}=150 \frac{\mathrm{mg}}{\mathrm{dl}}$, HDL cholesterol $\eta_{h}=50 \frac{\mathrm{mg}}{\mathrm{dl}}$ and WSS $\|\boldsymbol{\tau}\|=10 \%\|\overline{\boldsymbol{\tau}}\|$.

Table 4 Physiological ranges of LDL $\eta_{\ell}$ and HDL $\eta_{h}$ cholesterol concentrations in blood as well as wall shear stress exposure $\|\boldsymbol{\tau}\|$ in vivo.

\begin{tabular}{|c||c|c|c|}
\hline Parameter & Description & Range & Source \\
\hline \hline$\eta_{\ell}$ & Concentration of LDL cholesterol in blood & $10-3000 \frac{\mathrm{mg}}{\mathrm{dl}} *$ & 68,96,52] \\
$\eta_{h}$ & Concentration of HDL cholesterol in blood & $20-400 \frac{\mathrm{mg}}{\mathrm{dl}} *$ & 68,52,96] \\
$\|\boldsymbol{\tau}\|$ & WSS exposure & $0 \%-770 \%\|\overline{\boldsymbol{\tau}}\|$ & {$[93]$} \\
\hline
\end{tabular}

Parameters indicated by ${ }^{*}$ can be transformed into SI units using $\rho_{8}=4.36 \cdot 10^{17} \frac{1}{\mathrm{~g}}$ and $\rho_{13}=7.34 \cdot 10^{18} \frac{1}{\mathrm{~g}}$, cf. 92, Table 1 .

The remaining parameters of the spatially resolved model are given by the values parameterized in our previous work, see [92, Table 4. 


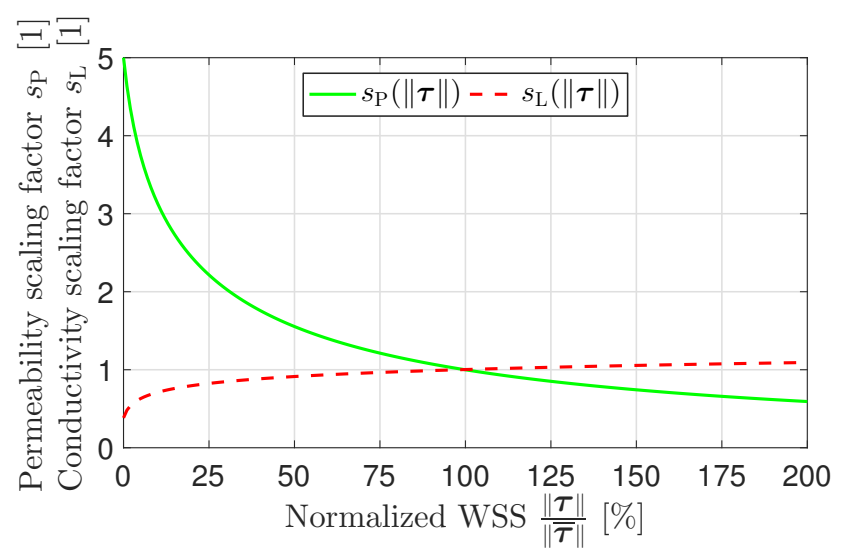

Fig. 4 Permeability scaling factor $s_{\mathrm{P}}(\|\boldsymbol{\tau}\|)$ and conductivity scaling factor $s_{\mathrm{L}}(\|\boldsymbol{\tau}\|)$ for varying normalized wall shear stresses $\frac{\|\boldsymbol{\tau}\|}{\|\overline{\boldsymbol{\tau}}\|}$.

\subsection{Lipoprotein Fluxes and Macrophage Recruitment}

The outcome of the spatially resolved model is determined by the rates of recruitment of native LDL $r_{\ell}(\ell, p,\|\boldsymbol{\tau}\|)$, modified LDL $r_{\tilde{\ell}}(\tilde{\ell}, p,\|\boldsymbol{\tau}\|)$ and HDL $r_{h}(h, p,\|\boldsymbol{\tau}\|)$ which characterize the physiology and diet of the host and the position of the individual plaque within the cardiovascular system. Here, the rates of recruitment depend on the three in vivo inputs, i.e. the blood concentrations of LDL $\eta_{\ell}$ and HDL $\eta_{h}$, and the WSS $\|\boldsymbol{\tau}\|$ that the model plaque is exposed to. The three in vivo inputs can - depending on the species of host, its predisposition and its diet - vary by several orders of magnitude (cf. Table 4) which results in qualitatively different predicted long-term outcomes.

The solute flux of lipoproteins through the endothelium is governed by the WSS-dependent second Kedem-Katchalsky equation and can be subdivided into diffusive and advective parts (cf. Eq. (4)). These parts are individually altered by the WSS-dependent PSF and CSF, see Figure 4. To investigate the influence of the WSS, the three submodels relative to their concentrations in blood, i.e., $\frac{r_{\ell}}{\eta_{\ell}}, \frac{r_{\tilde{Q}}}{\eta_{\ell}}$ and $\frac{r_{h}}{\eta_{h}}$ are estimated. Further, advective fractions of the total solute fluxes of native LDL $\frac{r_{\mathrm{Adv}, \ell}}{r_{\ell}}$, modified LDL $\frac{r_{\mathrm{Adv}, \tilde{\ell}}}{r_{\tilde{\ell}}}$ and HDL $\frac{r_{\mathrm{Adv}, h}}{r_{h}}$ are studied. To this end, we consider a pressure drop across the endothelium as measured by [89], i.e., $\Delta p_{\text {End }}=18 \mathrm{mmHg}$. Hence, different subendothelial pressures $p$ in between $75-90 \mathrm{mmHg}$ are considered. By analogy with the medial LDL concentrations measured in [36, 95], we use $\ell=\tilde{\ell}=7.2 \cdot 10^{-4} \eta_{\ell}$ and $h=7.2 \cdot 10^{-4} \eta_{h}$ as lipoprotein concentrations in the media.

The resulting relative fluxes $\frac{r_{i}}{\eta_{i}}$ and fractions of advective fluxes $\frac{r_{\mathrm{Adv}, i}}{r_{i}}$ of native LDL $\ell$, modified LDL $\tilde{\ell}$ and HDL $h$ are shown in Figure 5 . An overview of the estimated rates of recruitment of macrophages for various concentrations of modified LDL is given in Figure 6 . 
(a)

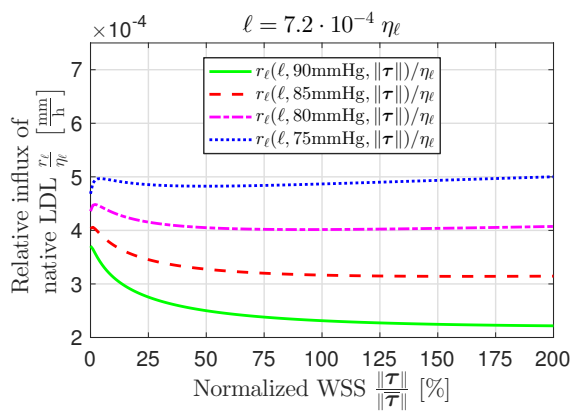

(c)

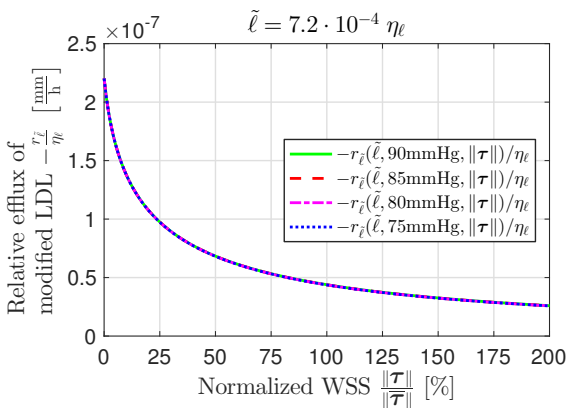

(e)

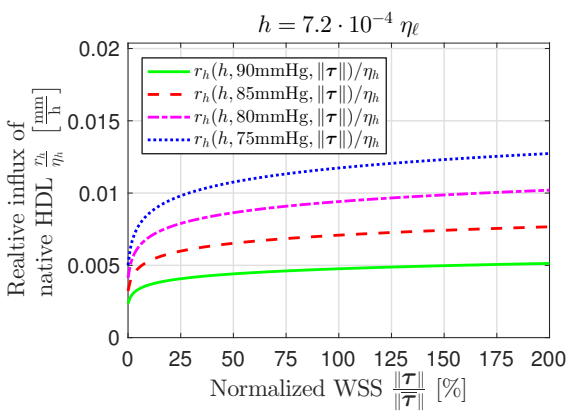

(b)

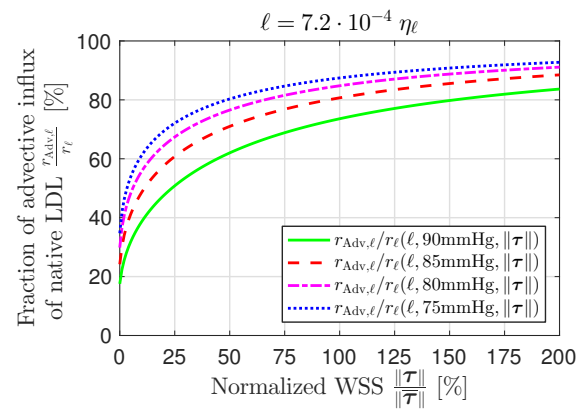

(d)

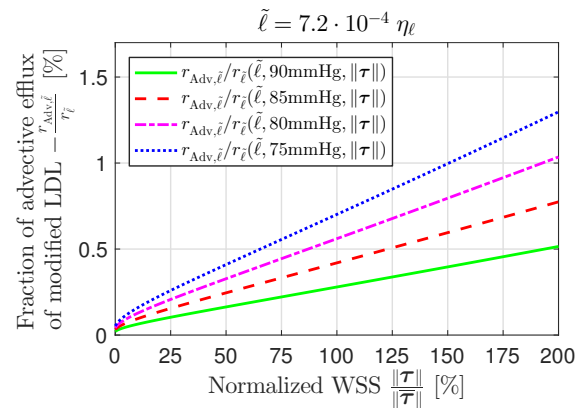

(f)

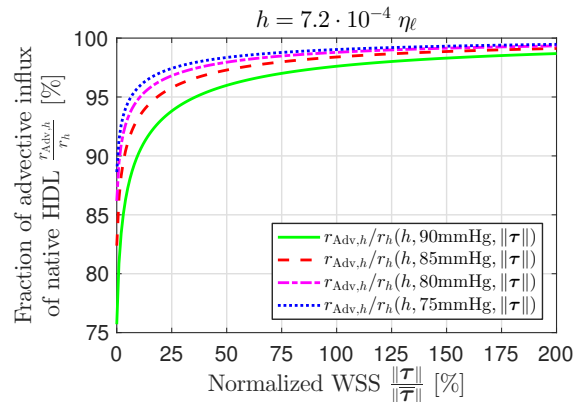

Fig. 5 Relative fluxes $\frac{r_{i}}{\eta_{i}}$ (a), (c) (e) and fractions of advective fluxes $\frac{r_{\mathrm{Adv}, i}}{r_{i}}$ (b), (d), (f) of native LDL (a) (b)), modified LDL [(c), (d)] and HDL $\stackrel{r_{i}}{(\mathrm{e})}$ (f) as predicted by the submodel of lipoprotein fluxes with varying normalized wall shear stresses $\frac{\|\boldsymbol{\tau}\|}{\|\bar{\tau}\|}$, various subendothelial pressures $p$ and prescribed subendothelial concentrations $\ell, \tilde{\ell}$ and $h$. 


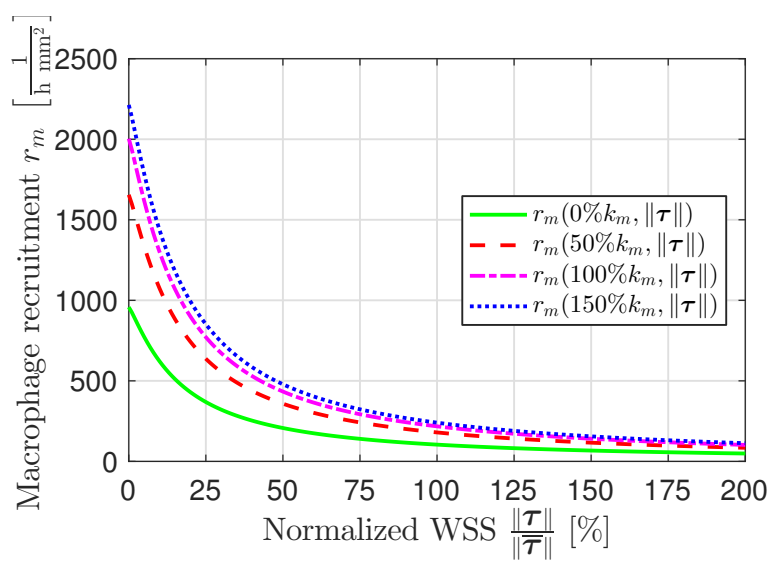

Fig. 6 Rate of recruitment of macrophages as predicted by the submodel of macrophage recruitment with varying normalized wall shear stresses $\frac{\|\boldsymbol{\tau}\|}{\|\bar{\tau}\|}$ and various concentrations of modified LDL $\tilde{\ell}$.

\subsection{Influence of Lipoprotein Transport within Artery Wall}

Within the artery wall LDL and HDL are transported by concentration gradientdriven diffusion and by advection due to the transmural flow which leads to spatially varying concentration profiles. The relative importance of the two transport mechanisms within the artery has not been investigated to date. The influence of diffusion on the lipoproteins' transport is assessed by a successive reduction of the order of magnitude of the effective diffusion coefficients $D_{\mathrm{Eff}, \ell}, D_{\mathrm{Eff}, \tilde{\ell}}$ and $D_{\mathrm{Eff}, h}$. These coefficients are reduced from large values that induce homogeneous concentrations, to their values as given in Table 2 that result in inhomogeneous concentration profiles along the radial direction. The importance of the advective transport of lipoproteins within the artery wall is then investigated by comparing the concentration profiles of the spatially resolved model, parameterized with various hindrance coefficients $K_{\ell}, K_{\tilde{\ell}}$ and $K_{h}$.

Denoting the concentration profile of native LDL along the $X$-axis by $\ell(X)$ (with $X \in[R, R+H]=[0.57 \mathrm{~mm}, 0.61 \mathrm{~mm}]$ ), the space-averaged concentration of native LDL $\bar{\ell}$ and its center of mass $\bar{X}_{\bar{\ell}}$ are given by

$$
\begin{gathered}
\bar{\ell}=\frac{1}{H} \int_{R}^{R+H} \ell(X) \mathrm{d} X, \\
\bar{X}_{\bar{\ell}}=\frac{1}{\bar{\ell}} \int_{R}^{R+H} X \ell(X) \mathrm{d} X .
\end{gathered}
$$


The average concentrations and centers of mass of the remaining species are computed analogously. The average concentrations of all species $\bar{\ell}, \ldots, \bar{m}$ along the $X$-axis which runs radially through the vessel wall and the position of their respective centers of mass $\bar{X}_{\bar{\ell}}, \ldots, \bar{X}_{\bar{m}}$ on the $X$-axis for various effective diffusion coefficients $D_{\mathrm{Eff}, \ell}, D_{\mathrm{Eff}, \tilde{\ell}}, D_{\mathrm{Eff}, h}$ and hindrance coefficients $K_{\ell}, K_{\tilde{\ell}}, K_{h}$ are given in Table 5. Moreover, the concentration profiles for various hindrance coefficients $K_{\ell}, K_{\tilde{\ell}}$ and $K_{h}$ are plotted in Figure 7 .

Table 5 Mean concentrations of native LDL $\bar{\ell}$, modified LDL $\overline{\tilde{\ell}}$, native HDL $\bar{h}$, intracellular free cholesterol $\frac{\bar{f}}{\bar{m}}$, intracellular cholesterol ester $\frac{\bar{f}}{\bar{m}}$ and macrophages $\bar{m}$ and their centers of mass $\bar{X}_{\bar{i}}$ for varying effective diffusivities $D_{\mathrm{Eff}, \ell}\left(=D_{\mathrm{Eff}, \tilde{\ell}}=\frac{1}{2.33} D_{\mathrm{Eff}, h}\right)$ and hindrance coefficients $K_{\ell}$ $\left(=K_{\tilde{\ell}}=\frac{1}{5.36} K_{h}\right)$ of native LDL at time $t=100$ weeks.

\begin{tabular}{|c|c|c|c|c|c|c|c|c|c|c|c|c|c|c|}
\hline \multicolumn{2}{|c|}{ Parameter setup } & \multicolumn{4}{|c|}{\begin{tabular}{|l|l|} 
Mean concentration $\frac{1}{\mathrm{~mm}^{3}}$ \\
\end{tabular}} & \multicolumn{2}{|l|}{ or $[1]$} & \multicolumn{6}{|c|}{ Center of mass [\%] * } & \multirow[t]{2}{*}{ Plot } \\
\hline$\frac{1}{1.26} \cdot D_{\mathrm{Eff}, \ell}$ & $K_{\ell}$ & $10^{-6} \cdot \bar{\ell}$ & $10^{-7} \cdot \overline{\tilde{\ell}}$ & $10^{-7} \cdot \bar{h}$ & $10^{-10} \cdot \frac{\bar{f}}{m}$ & $10^{-11} \cdot \frac{b}{\bar{m}}$ & $10^{-7} \cdot \bar{m}$ & $\bar{X}_{\bar{\ell}}$ & $\bar{X}_{\bar{\ell}}$ & $\bar{X}_{\bar{n}}$ & $\bar{X}_{\frac{\bar{I}}{\bar{T}}}$ & $\bar{X}_{\frac{\bar{b}}{\bar{m}}}$ & $\bar{X}_{\bar{m}}$ & \\
\hline $10^{2}$ & $\overline{0.12 * *}$ & 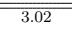 & 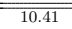 & 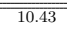 & 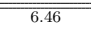 & 2.33 & 3.37 & $\overline{50}$ & $\overline{50}$ & 50 & 50 & 50 & 50 & - \\
\hline $10^{0}$ & 0.12 ** & 2.6 & 9.73 & & & & & 41.3 & 50.9 & 40.8 & 51.4 & 54.0 & 48.5 & \\
\hline $10^{-1}$ & $0.12^{* *}$ & 2.66 & 90 & 9.04 & 6.27 & & 3.27 & 18.6 & 48.9 & 17.9 & 51.3 & 54.1 & 48.4 & - \\
\hline $10^{-2 * *}$ & & 2.58 & & & 6.39 & & & 5.51 & 37.2 & 5.32 & 51.3 & 54.1 & 48.3 & \\
\hline $10^{-2 * *}$ & $0.12 * *$ & 2.58 & 8.84 & 8. & 6.39 & 2.75 & & 5.52 & 37.3 & 5.33 & 51.3 & 54.1 & 48.3 & Fig. 7 \\
\hline $10^{-2 * *}$ & 1 & 2.58 & 8.94 & 8.79 & 6.39 & 2.75 & 3.19 & 5.55 & 38.1 & 5.33 & 51.3 & 54.1 & 48.3 & Fig. \\
\hline $10^{-2 * *}$ & 5 & 2.59 & 9.33 & 8.81 & 6.39 & 2.74 & 3.18 & 5.71 & 41.5 & 5.40 & 51.3 & 54.1 & 48.3 & Fig. \\
\hline $10^{-2 * *}$ & 25 & $\begin{array}{l}2.63 \\
\end{array}$ & 9.70 & 9.04 & 6.39 & 2.72 & $\begin{array}{l}3.11 \\
\end{array}$ & 6.63 & 55.6 & 5.79 & 51.3 & 54.1 & 48.3 & Fig. \\
\hline
\end{tabular}

* $0 \%$ and $100 \%$ correspond to the endothelium and media-adventitia boundary, respectively.

** Corresponds to the physiological parameter from Table 2

\subsection{Influence of Inhomogeneous WSS Distribution}

In addition to diffusion and advection, the concentration profiles are locally altered in vivo by non-uniform recruitment rates of LDL, HDL and macrophages through the endothelium, e.g., induced by spatially varying WSS pattern due to disturbed flows. Regional differences in the recruitment rates can lead to the local formation of plaques whereas other sites remain healthy at the same time. Hence, we solve and analyse the spatially resolved model with a prescribed synthetic inhomogeneous normalized WSS distribution as shown in Figure 3 . In accordance with WSS patterns in the literature [93, 19, 25, 87], the prescribed WSS pattern has a sharp transition from low $(10 \%\|\bar{\tau}\|)$ to high $(100 \%\|\bar{\tau}\|)$ WSS.

The steady-state concentration profiles of the species $\hat{\ell}(X), \ldots, \hat{m}(X)$ in the spatially resolved model with prescribed inhomogeneous WSS (cf. Figure 3) on the negative $X$-axis (where $\|\boldsymbol{\tau}\|=100 \%\|\overline{\boldsymbol{\tau}}\|$ ) and the positive $X$-axis (where $\|\boldsymbol{\tau}\|=10 \%\|\overline{\boldsymbol{\tau}}\|$ ) are plotted in Figure 8. An overview of the spatial distribution of the steady-state concentrations of modified LDL $\widehat{\tilde{\ell}}$, intracellular 


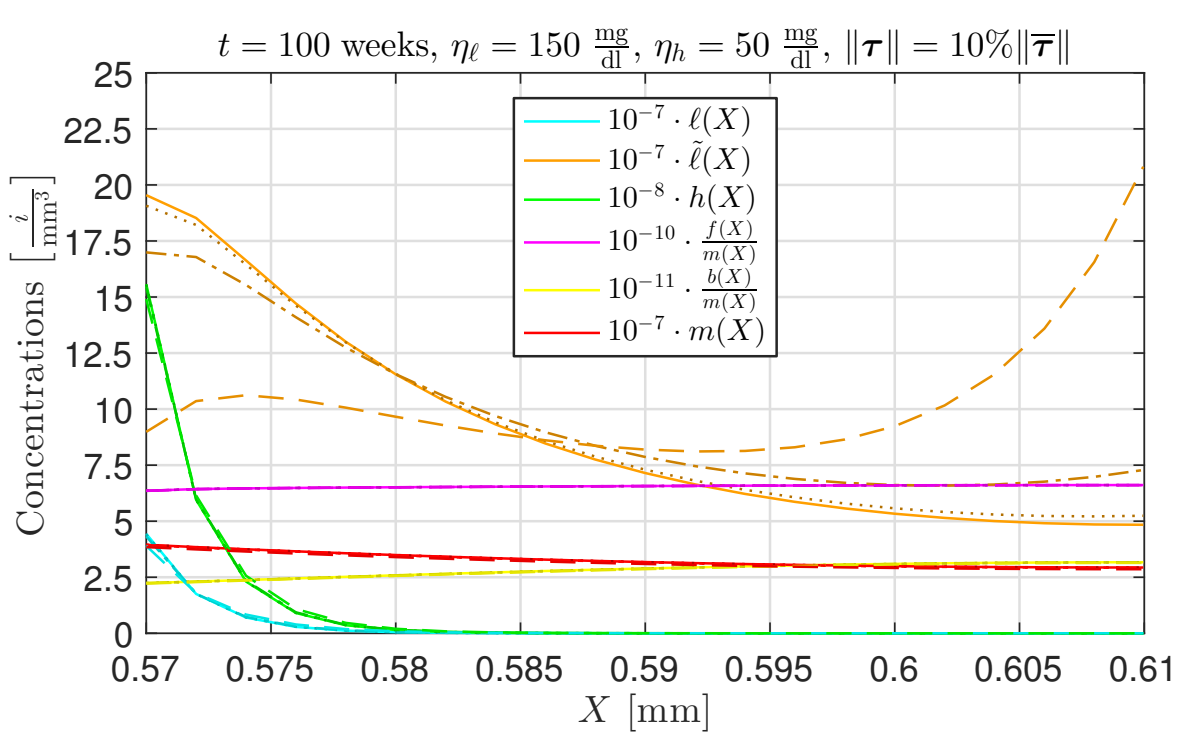

Fig. 7 Concentration profiles of native LDL $\ell$, modified LDL $\tilde{\ell}$, native HDL $h$, intracellular free cholesterol $\frac{f}{m}$, intracellular cholesterol ester $\frac{b}{m}$ and macrophages $m$ at time $t=100$ weeks plotted on the $X$-axis. Hindrance coefficients $K_{\ell}=0.12$ (solid lines), $K_{\ell}=1$ (dotted lines), $K_{\ell}=5$ (dash-dotted lines) or $K_{\ell}=25$ (dash lines).

free cholesterol $\frac{\widehat{f}}{\hat{m}}$, intracellular cholesterol ester $\frac{\widehat{b}}{\widehat{m}}$ and macrophages $\widehat{m}$ at the endothelial boundary $\Gamma_{\text {End }}$ are shown in Figure 9 
(a)

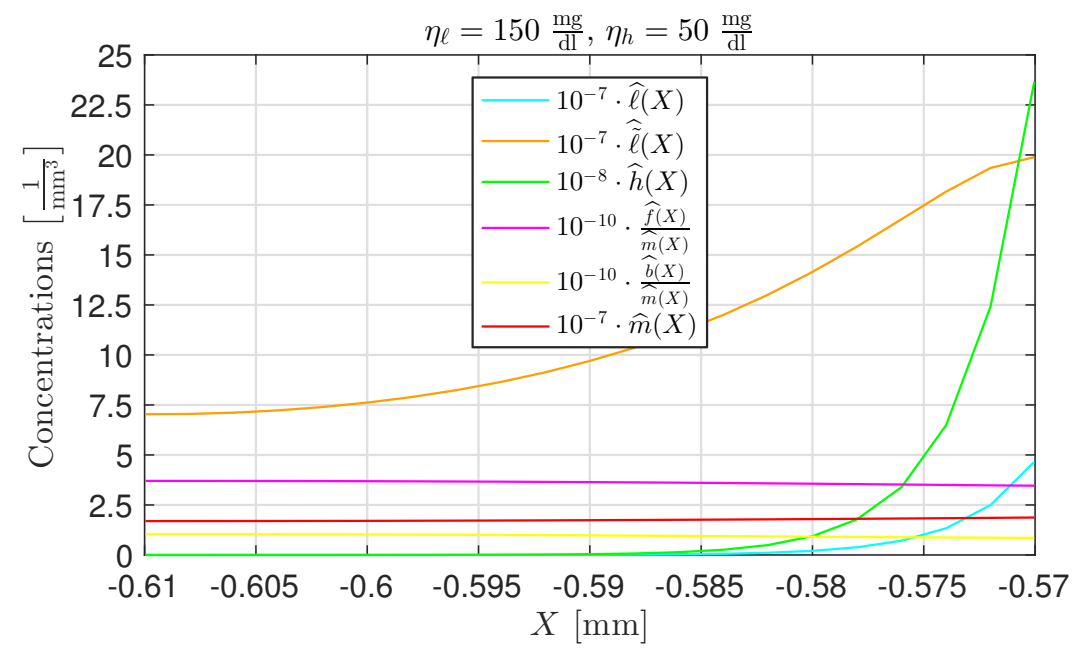

(b)

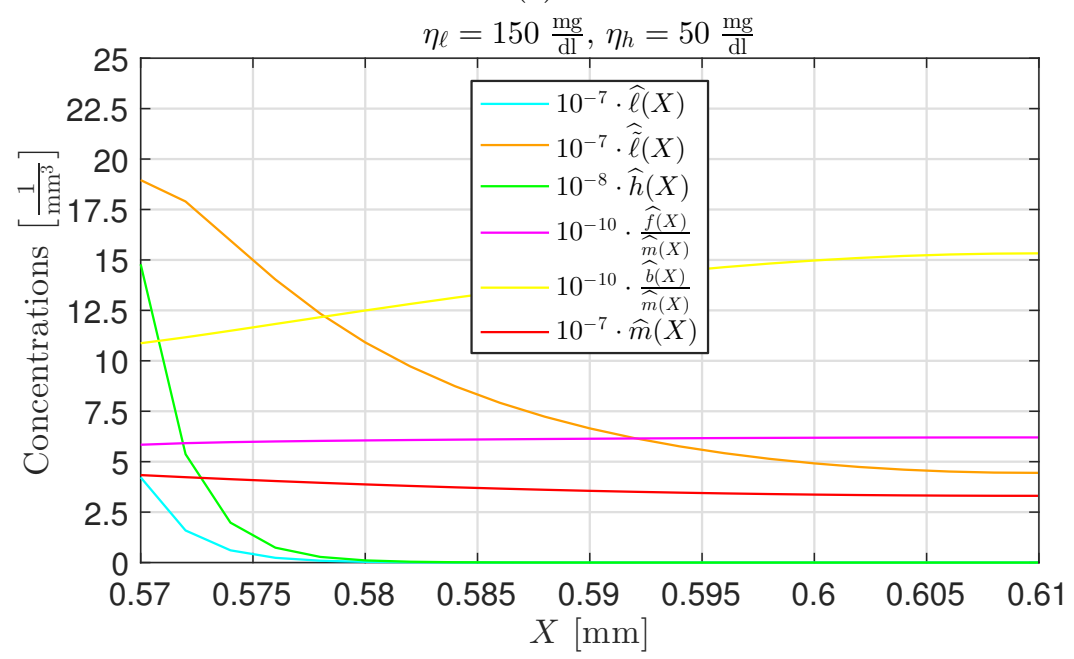

Fig. 8 Steady-state concentration profiles of native LDL $\widehat{\ell}$, modified LDL $\widehat{\tilde{\ell}}$, native HDL $\widehat{h}$, intracellular free cholesterol $\frac{\widehat{f}}{\widehat{m}}$, intracellular cholesterol ester $\frac{\widehat{b}}{\widehat{m}}$ and macrophages $\widehat{m}$ plotted on the (a) positive $X$-axis and (b) negative $X$-axis. Blood cholesterol concentrations $\eta_{\ell}=150 \frac{\mathrm{mg}}{\mathrm{dl}}, \eta_{h}=50 \frac{\mathrm{mg}}{\mathrm{dl}}$ and nonhomogeneous WSS $\|\boldsymbol{\tau}\|$ given by Figure 3 . 
(a)

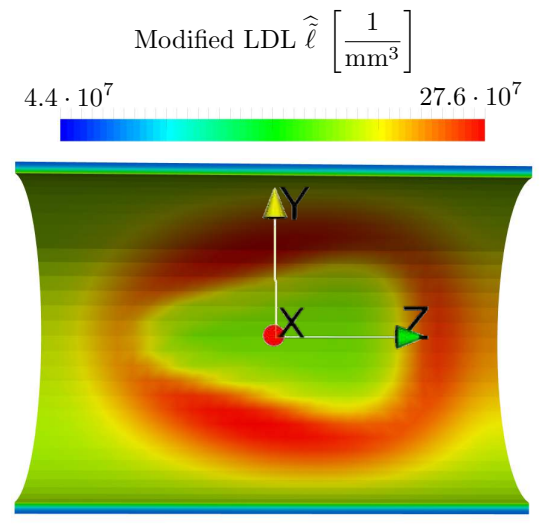

(c)

Intercellular cholesterol ester $\frac{\widehat{b}}{\widehat{m}}\left[\frac{1}{\mathrm{~mm}^{3}}\right]$ $0.8 \cdot 10^{10}$ $28.1 \cdot 10^{10}$
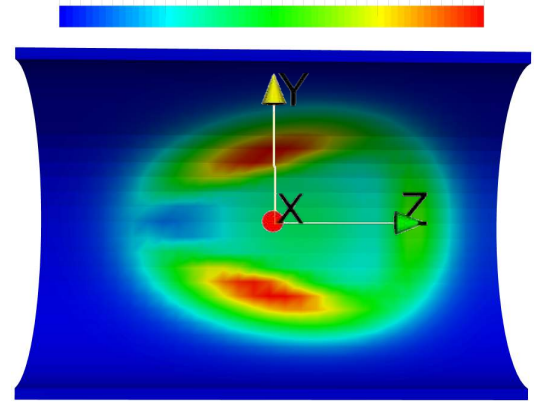

(b)

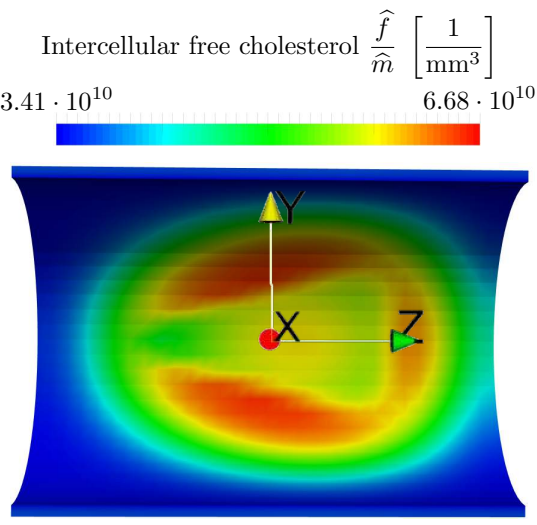

(d)

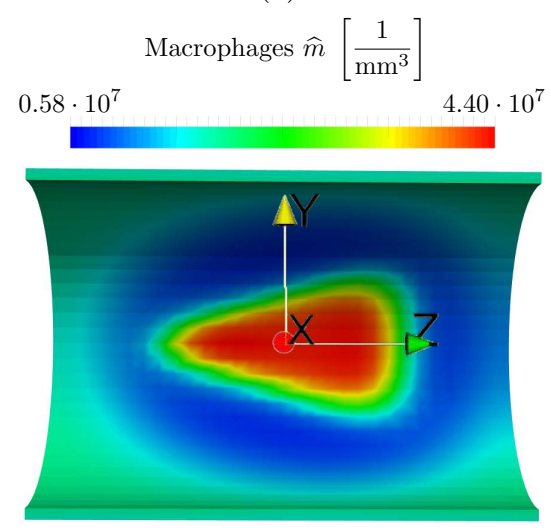

Fig. 9 Spatial distributions of steady-state concentration of (a) modified LDL $\widehat{\tilde{\ell}}$, (b) intracellular free cholesterol $\frac{\widehat{f}}{\hat{m}}$, (c) intracellular cholesterol ester $\frac{\widehat{b}}{\widehat{m}}$ and (d) macrophages $\widehat{m}$. Blood cholesterol concentrations $\eta_{\ell}=150 \frac{\mathrm{mg}}{\mathrm{dl}}, \eta_{h}=50 \frac{\mathrm{mIg}}{\mathrm{dl}}$ and non-homogeneous WSS $\frac{\|\boldsymbol{\tau}\|}{\|\overline{\boldsymbol{\tau}}\|}$ as given in Figure 3

\section{Discussion}

In this paper we have developed a quantified spatially resolved model of key species of early atherosclerosis. A complete parameter set for the spatially resolved model was derived from existing experimental data (cf. Tables 2,3 and (4). The influence of uncertainties due to the parameters' origins, different animal models used in the experiments, or measurement errors is inverstigated in Appendix B.2. 
The influence of disturbed blood flow on the recruitment of monocytes in vivo requires further focus in future experimental work. Only very few experimental results investigate non-constant flow patterns or assess the influence of complex flows to the endothelium 39. As commonly done, the time-averaged WSS $\|\boldsymbol{\tau}\|$ were employed as indicator for disturbed blood flow [7, 48. A more rigorous mathematical approach would introduce effects of the pulsatile flow to provide a more accurate estimate of the influence of the blood flow on the endothelium. Further experimental results are needed, however, to justify such an additional mathematical sophistication.

The submodel for the recruitment of macrophages was derived and quantified from experimental results in 42] which investigated the adherence of monocytes on modified LDL-activated endothelial cells. To this end, we assumed that WSSdependent adherence to the endothelium is the determining step in monocyte migration into the intima and used this assumption to estimate monocyte migration rates from adherence data. No direct quantitative data exist that allow to quantify the actual rate of monocyte recruitment into the intima.

Three submodels based on the equations of Kedem and Katchalsky were developed and parameterized to assess the transport of native LDL $r_{\ell}$, modified LDL $r_{\tilde{\ell}}$ and native HDL $r_{h}$ through the endothelium. The total flux of lipoproteins can be subdivided into diffusive and advective parts which vary depending on the WSS $\|\boldsymbol{\tau}\|$ due to the PSF and CSF that we have employed (cf. Figure 4 ). As a consequence, the total fluxes and the fractions of the advective fluxes of native LDL, modified LDL and native HDL significantly differ depending on the WSS. While the diffusive efflux determines the total efflux of modified LDL $r_{\tilde{\ell}}$ (cf. Figure $5(\mathrm{~d})$ for all relevant WSS and medial pressures, the total influx of native HDL $r_{h}$ is dominated by the advective influx (cf. Figure $5(\mathrm{f})$ ). The situation with advective influx of native LDL is ambiguous as advective influx is dominant for low WSS regimes but not significant at high WSS (cf. Figure $5(\mathrm{~b})]$. The results support the idea that the advective influx of native LDL and HDL through the endothelium significantly determines their total influx rate. It follows that a porous medium-like approach which considers the pressure gradient-driven advective transmural flow through the endothelium for estimation the recruitment rates of lipoproteins is inevitable.

The total efflux of modified LDL $r_{\tilde{\ell}}$ is more than three orders of magnitude smaller compared to the fluxes of native LDL and HDL (cf. Figures 5) 5 (c) and $5(\mathrm{e})$. Thus, the efflux of modified LDL from the intima is negligible. Due to their smaller particle size, the flux of native HDL into the intima is approximately 10 to 25 times larger than the influx of native LDL (see Figures $5(\mathrm{a})$ and $5(\mathrm{e})$. It is important to note, however, that the influx of native HDL increases with increasing WSS while the influx of native LDL decreases. Together with a decreased recruitment of macrophages under high WSS (see Figure 6), the stability of model plaques is changed from vulnerable to stable with increasing WSS [92]. The findings further support that the WSS and therefore the location of an early plaque within the cardiovascular system already decisively determines its type, i.e., if it is progression-prone or progression-resistant, cf. 82. 
Besides the flow-dependent and thus non-modifiable WSS, the cholesterol concentrations of LDL $\eta_{\ell}$ and HDL $\eta_{h}$ play an important role in atherogenesis. They can vary by several orders of magnitude depending on the physiology and diet of a plaque's host (cf. Table 4 ) and induce qualitatively different long-term outcomes of model plaques. A risk analysis performed by Hao and Friedman [34 shows that the effect of a drop in the availability of native HDL is much more severe than of LDL. This aligns with the results of our stability analysis in Appendix B.3. It also shows, however, that the most crucial determinant of plaque stability is the WSS $\|\boldsymbol{\tau}\|$ (see Figure 12). While the blood cholesterol concentration of HDL $\eta_{h}$ also has a major impact, only very high blood cholesterol concentrations of LDL $\eta_{\ell}$ significantly influence the long-term model plaque outcome. However, such LDL cholesterol concentrations usually do not occur in vivo unless they are enforced by experimental interventions, e.g., by putting genetically modified mice on a high-fat diet. The results in Appendix B.3. Figure 12 support the hypothesis that the natural resistance of mice to atherosclerosis may be due to their generally high HDL and low LDL blood cholesterol profiles [52,99. Further, for the common range of blood cholesterol concentrations in humans [68, plaques at locations with a WSS exposure below approximately $\|\boldsymbol{\tau}\|=20 \%\|\overline{\boldsymbol{\tau}}\|=0.2$ Pa (cf. Table 3 ) must be considered as progression-prone plaques, i.e., as plaques of type IIa in the classification by the American Heart Association 82.

Darcy's law in combination with the first Kedem-Katchalsky equation was used to develop a model of the blood plasma pressure and pressure gradientdriven transmural flow in the artery wall. An average transmural filtration velocity of $\|\boldsymbol{u}\|=6.47 \cdot 10^{-2} \frac{\mathrm{mm}}{\mathrm{h}}$ is estimated by the spatially resolved model which is in very good agreement with experimental and computational results in the literature $[63,29,103,74]$. Using this average velocity value, the Reynolds number $R e$ of the transmural filtration flow is calculated as

$$
R e=\frac{\varrho\|\boldsymbol{u}\| H}{\mu}=1.05 \cdot 10^{-6} .
$$

The Reynolds number is $R e \ll 1$, and so the application of Darcy's law results in a good approximation to the viscous transmural flow within aortic tissue [50,3,29. Using Darcy's law, an intima-media pressure drop of $\Delta p_{\text {Med }}=52.4 \mathrm{mmHg}=7.0 \cdot 10^{3} \mathrm{~Pa}$ is estimated which is in very good agreement to experimental results in 89 .

Crucial parameters that were not covered by the sensitivity analysis in Appendix B.2 are the effective diffusivities of native LDL, modified LDL and HDL. In the literature, there is no agreement on the value of $D_{\mathrm{Eff}, \ell}$. In contrast, almost all orders of magnitude in between the range of $D_{\mathrm{Eff}, \ell}=1.2 \cdot 10^{2} \frac{\mathrm{mm}^{2}}{\mathrm{~h}}$ 30] to $D_{\mathrm{Eff}, \ell}=1.8 \cdot 10^{-4} \frac{\mathrm{mm}^{2}}{\mathrm{~h}}$ [103] are found. In this work, based on an extensive literature review, we use a frequently found intermediate value $D_{\mathrm{Eff}, \ell}=$ $1.26 \cdot 10^{-2} \frac{\mathrm{mm}^{2}}{\mathrm{~h}}[94,86,10]$. A comparison of the computational results shows that effective diffusion coefficients in the order of magnitude of $\mathcal{O}\left(10^{2} \frac{\mathrm{mm}^{2}}{\mathrm{~h}}\right)$ result in concentrations without spatial variations. With decreasing diffusivities, 
the concentrations of lipoproteins along the radial direction change from homogeneous concentrations to concentration profiles with steep gradients at the endothelium, see Table 5 and Figure 7 In contrast, the density of macrophages and their intracellular free and esterified cholesterol contents remain more or less homogeneously. Even though their centers of mass shift by the reduction of the diffusivities, the average concentrations of all species $\bar{\ell}, \bar{\ell}, \bar{h}, \bar{f}, \bar{b}, \bar{m}$ are only mildly affected $(<18 \%)$ when diffusivities are reduced (cf. Table 5 ).

The variation of the hindrance coefficients $K_{\ell}, K_{\tilde{\ell}}$ and $K_{h}$ shows that species' concentrations are barely affected $(<10 \%)$ by the advective transport within the artery wall, see Table 5 and Figure 7 . If the transmural flow in the artery wall is artificially increased by high hindrance coefficients, only the concentration profile of modified LDL is significantly altered, while the remaining concentration profiles are barely affected. As hindrance coefficients are in general $0 \leq K_{i} \leq 1$, such fast advective transport is only achieved by an approximately 100 times higher transmural velocity of $\|\boldsymbol{u}\|$. This is far beyond velocities reported in the literature and the advective transport cannot explain the increase of the LDL profile toward the media-adventitia boundary as observable in vivo [63,20,95,5]. The driving mechanism behind this observation thus remains unknown. In total, the results indicate that the influence of the advective transport due to the transmural flow inside the artery wall is negligible. Still, the model of the transmural flow cannot completely be neglected as it also determines the subendothelial pressure $p$ and thus significantly alters the total lipoprotein fluxes as discussed before.

Important spatial phenomena occur as a result of inhomogeneous boundary conditions (see Figures 8 and 9). The steady-state concentration profiles of the spatially resolved model with prescribed inhomogeneous WSS pattern (cf. Figure 3) along the radial directions are qualitatively different. The negative $X$-axis corresponds to a region exposed to high WSS $\|\boldsymbol{\tau}\|=100 \%\|\overline{\boldsymbol{\tau}}\|$. Thus, the atherosclerotic processes remains non-critical with a low density of macrophages $\widehat{m}$ with low intracellular free cholesterol $\frac{\widehat{f}}{\hat{m}}$ and almost no intracellular cholesterol ester $\frac{\widehat{b}}{\widehat{m}}$ (cf. Figure $g(a)$. The positive $X$-axis corresponds to a region exposed to low WSS $\|\boldsymbol{\tau}\|=10 \%\|\bar{\tau}\|$. As a consequence, along the negative $X$-axis characterizations of an vulnerable plaque such as a high density of macrophages $\widehat{m}$ with high intracellular free cholesterol $\frac{\widehat{f}}{\hat{m}}$ and high intracellular cholesterol ester $\frac{\widehat{b}}{\widehat{m}}$ content can be found (cf. Figure $g(\mathrm{~b})$.

Due to the diffusion of all species from the strongly atherosclerotic regions into the surrounding, non-atherosclerotic regions, the area of the model plaque is increased compared to the low WSS area (cf. Figures 3 and 9). The region surrounding the area of low WSS has higher concentrations of intracellular free and esterified cholesterol than the central part of the plaque, although it has substantially fewer macrophages and is less inflamed by lower concentrations of modified LDL (cf. Figure 9). This effect may, however, be reduced by adding a macrophage chemotactic response to modified LDL [12,11]. Macrophages at the center of the lesion are able to preserve a stable concentration of 
modified LDL $\widehat{\tilde{\ell}}$ in the tissue, comparable to non-atherosclerotic sites (see Figure $\mathrm{g}(\mathrm{a})$. However, this comes at the cost of an increased concentration of intracellular free cholesterol $\frac{\widehat{f}}{\hat{m}}$ and cholesterol ester $\frac{\widehat{b}}{\widehat{m}}$ inside macrophages rendering them more susceptible to apoptosis and becoming necrotic foam cells. Hence, plaques at the low WSS region and the surrounding tissue must be considered progression-prone, i.e., as type IIa [82.

The spatially resolved model of key species of early atherosclerosis that we have developed is able to predict the stability of early model plaques based on the blood cholesterol concentrations of LDL and HDL and the WSS it is exposed to. In our model, macrophages only undergo recruitment and apoptosis which is valid only for early stages of atherosclerosis. As discussed in detail in 92, in the future, the fate of macrophages must be enriched by emigration, proliferation and necrosis to study intermediate and advanced plaques.

Only a simplified geometrical setup was investigated here, where an synthetic WSS distribution was prescribed. To consider patient-specific anatomies, the spatially resolved model can be embedded into a multiphysics model 93. using a suitable multiscale in time strategy [27,47,94]. For a patient-specific simulation, the time-averaged local WSS, luminal pressure and cholesterol concentrations from a pulsatile fluid or fluid-structure interaction simulation can be used 93. to find locally varying physiological lipoprotein fluxes and macrophage recruitments. These can then be used to create a personalized model of a particular plaque in its early stages.

\section{Conclusion}

This paper presents a quantified spatially resolved mathematical model of early atherosclerosis. Based on basic continuum mechanical laws, we have formulated a quantitative model that naturally brings together Darcy's law, our quantitative ODE model developed in [92] as well as submodels of the recruitment of macrophages and fluxes of lipoproteins that have been parameterized using existing experimental and theoretical results.

The model allows us to differentiate between progression-prone and progression-resistant plaques based on three measurable or computable in vivo inputs - the WSS that a plaque is exposed to, and blood cholesterol concentrations of LDL and HDL. The model indicates that the influence of the advective transport of lipoproteins through the activated endothelium by the transmural flow is decisive, while the advective transport within the artery wall is negligible. Further, the model suggests that regions in arteries with an approximate WSS exposure below $20 \%$ of the average WSS and their surroundings must be considered as potential regions where progression-prone atherosclerotic plaques develop.

Acknowledgements Michael W. Gee and Moritz P. Thon gratefully acknowledge the support and funding given by the International Graduate School of Science and Engineering of the TUM under project BioMat01, A Multiscale Model of Atherosclerosis and the Leibniz 
Rechenzentrum München of the Bavarian Academy of Sciences under contract number pr48ta. Mary R. Myerscough acknowledges support from an Australian Research Council Discovery Project Grant.

Conflict of Interest All authors declare that no conflicts of interest exist.

\section{Appendix A Quantification of Model Parameters}

In this section, a complete set of parameters for the spatially resolved model in SI units $\mathrm{mm}, \mathrm{g}$ and $\mathrm{h}$ is derived. Due to a lack of uniform experimental data we do not distinguished between experimental results gained from the study of different animal models. As the media is the predominant part of the intima-media domain [28, 37, 103, 74, we use parameters that correspond to the media.

Appendix A.1 Macrophage Related Parameters

The parameters of the submodel of monocyte adhesion least-squares fitted to the experimental results by Jeng [42] are

$$
\begin{aligned}
P_{m} & =443.17 \frac{1}{\mathrm{~h} 0.1452 \mathrm{~mm}^{2}}=3052.13 \frac{1}{\mathrm{~h} \mathrm{~mm}^{2}}, \quad \delta_{m}=68.62 \%, \\
k_{m} & =7.09 \cdot 10^{-3} \frac{\mu \mathrm{g} \text { lipid protein }}{\mathrm{ml}} \widehat{=} 7.38 \cdot 10^{6} \frac{1}{\mathrm{~mm}^{3}}, \quad \delta_{\tau}=40.22 \%, \\
\xi_{\tau} & =1.95 \frac{\mathrm{dyn}}{\mathrm{cm}^{2}}=1.95 \cdot 10^{-1} \mathrm{~Pa}, \quad \nu_{\tau}=1.18
\end{aligned}
$$

where all values are given in the unit of the experiment and SI units. The conversion of $k_{m}$ from $\mu \mathrm{g}$ lipid protein to SI units is performed using $\rho_{10}=$ $1.04 \cdot 10^{9} \frac{1}{\mu \mathrm{g} \text { lipid protein }}$ from [92], Table 1. An overview of the least-squares fits is given in Figure 10|(a). Since intracellular free and esterified cholesterol can only diffuse within macrophages, the macrophages' effective diffusion coefficient is also employed for the cholesterols, i.e., $D_{\mathrm{Eff}, m}=D_{\mathrm{Eff}, f}=D_{\mathrm{Eff}, b}=$ $3.6 \cdot 10^{-6} \frac{\mathrm{mm}^{2}}{\mathrm{~h}}$, cf. [15, 30].

\section{Appendix A.2 LDL Related Parameters}

The influx of native LDL through the endothelium described by the second Kedem-Katchalsky equation (cf. Eq. (4)) is well-studied and thus the required parameters are available in the literature. In contrast, the flux of modified LDL and native HDL are scant investigated. Due to its origin and equal size, it is convenient to use the same parameters for native and modified LDL. In contrast to native LDL, however, the concentration of modified LDL in blood is low [23] and therefore we use $\eta_{\tilde{\ell}}=0$. 
(a)

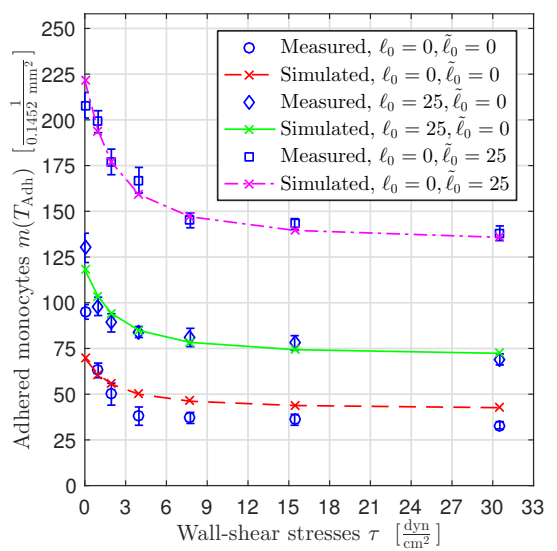

(c)

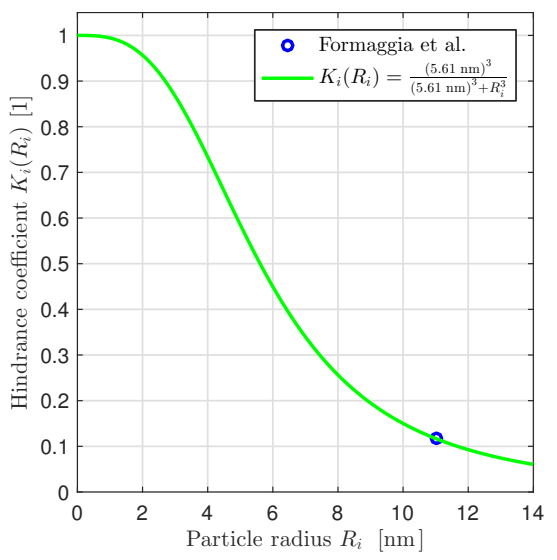

(b)

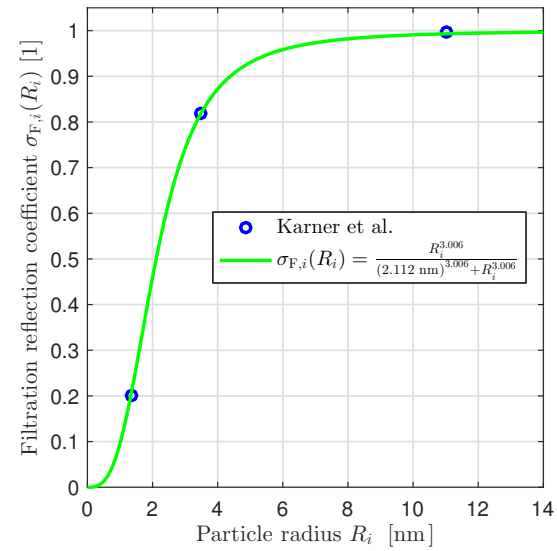

(d)

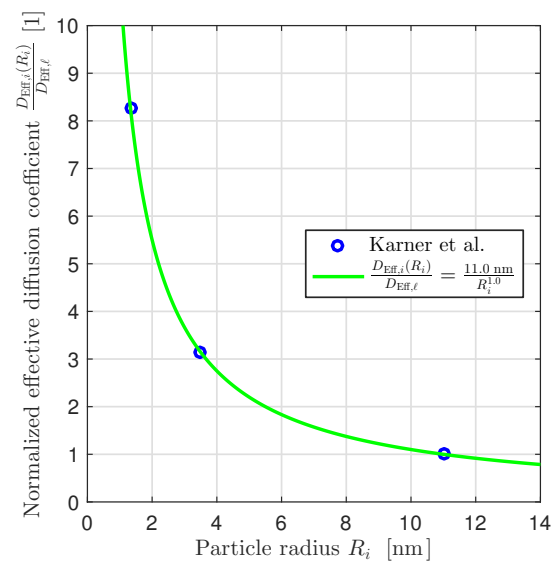

Fig. 10 Least-squares fits of (a) simulated monocyte adhesion $m\left(T_{\mathrm{Adh}}\right)$ to experimental results by Jeng et al. [42], Table 1, (b) filtration reflection coefficient $\sigma_{\mathrm{F}, i}$ to results by Karner et al. 44, Table 3, (c) hindrance coefficient $K_{i}$ to result by Formaggia et al. [29] and (d) effective diffusion coefficient $D_{\mathrm{Eff}, i}$ by Karner et al. 44, Table 2 .

The weighting factors $\omega_{\ell}, \omega_{\tilde{\ell}}$ and $\omega_{h}$ for the average concentrations of native LDL, modified LDL and native HDL within the endothelium layer are estimated as proposed by Formaggia et al. [29]. The one-dimensional advection-diffusion equation is solved and volume-averaged over the domain of the endothelium. It follows that

$$
\omega_{i}=\frac{\exp \left(P e_{i}\right)}{\exp \left(P e_{i}\right)-1}-\frac{1}{P e_{i}},
$$


where $P e_{i}$ is the Péclet number of species $i$ within the endothelium given by

$$
P e_{i}=\frac{\|\boldsymbol{u}\|}{P_{i}} .
$$

Here, $\|\boldsymbol{u}\|$ and $P_{i}$ are the transmural filtration velocity and the diffusive permeability of species $i$, respectively. Using $\|\boldsymbol{u}\|=6.41 \cdot 10^{-2} \frac{\mathrm{mm}}{\mathrm{h}}$ [29, 103,63 , and $P_{\ell}=P_{\tilde{\ell}}=6.12 \cdot 10^{-5} \frac{\mathrm{mm}}{\mathrm{h}}$ [95, 103] results in $P e_{\ell}=P e_{\tilde{\ell}}=1.05 \cdot 10^{3}$ and weighting factors $\omega_{\ell}=\omega_{\tilde{\ell}}=9.99 \cdot 10^{-1}$.

To estimate the initial rate of recruitment of macrophages $r_{m}\left(\tilde{\ell}_{0},\|\boldsymbol{\tau}\|\right)$, we use an initial concentration of modified LDL $\tilde{\ell}_{0}=7.2 \cdot 10^{-4} \eta_{\ell}$ based on the experimental results in [36,95].

\section{Appendix A.3 HDL Related Parameters}

HDL is a smaller particle than LDL. More precisely, LDL and HDL have radii of $R_{\ell}=11.0 \mathrm{~nm}$ [44] and $R_{h}=4.72 \mathrm{~nm}$ [46], respectively. As a consequence, HDL has approximately an 1.87 times higher diffusive permeability compared to LDL, i.e., $P_{h}=1.87 P_{\ell}=1.14 \cdot 10^{-4} \frac{\mathrm{mm}}{\mathrm{h}}$ [83, 95]. Together with Eqs. 23. and 24, it follows the weighting factor $\omega_{h}=9.98 \cdot 10^{-1}$ for the average concentrations of native HDL in the endothelium layer.

Karner et al. 44 investigated the transport rates of ADP, albumin and LDL through the endothelium and the internal elastic lamina. As ADP and albumin have a smaller and LDL has a larger radius compared to HDL, their filtration reflection coefficients are used to estimate the filtration reflection coefficient $\sigma_{\mathrm{F}, h}$ of HDL. We performed a least-squares fit of the filtration reflection coefficient as given in [44, Table 3 to a saturating function in the particle radius $R_{i}$ of the form $\sigma_{\mathrm{F}, i}\left(R_{i}\right)=\frac{R_{i}^{a}}{b^{a}+R_{i}^{a}}$. The least-squares fit yields $a=3.006$ and $b=2.112 \mathrm{~nm}$ and so

$$
\sigma_{\mathrm{F}, i}\left(R_{i}\right)=\frac{R_{i}^{3.006}}{(2.112 \mathrm{~nm})^{3.006}+R_{i}^{3.006}},
$$

which is visualized in Figure 10(b) The filtration reflection coefficient scales approximately with the volume of particles and for HDL this gives $\sigma_{\mathrm{F}, h}=9.18 \cdot 10^{-1}$.

The hindrance coefficient of LDL in the media is given by $K_{\ell}=K_{\tilde{\ell}}=0.117$ 29 70, respectively. Assuming that the hindrance coefficient scales with the volume of particles (in consistency to the filtration reflection coefficient) and a saturating kinetic (in general it holds $0 \leq K_{i}\left(R_{i}\right) \leq 1$ ), it follows

$$
K_{i}\left(R_{i}\right)=\frac{(5.61 \mathrm{~nm})^{3}}{(5.61 \mathrm{~nm})^{3}+R_{i}^{3}},
$$

which is plotted Figure 10(c) Accordingly, the hindrance coefficient of HDL is estimated to $K_{h}=6.27 \cdot 10^{-1}$.

An additional unknown parameter is the effective diffusion coefficient of HDL $D_{\mathrm{Eff}, h}$ within the artery wall. The effective diffusivity of LDL is measured 
as $D_{\mathrm{Eff}, \ell}=D_{\mathrm{Eff}, \tilde{\ell}}=1.26 \cdot 10^{-2} \frac{\mathrm{mm}^{2}}{\mathrm{~h}}$ 94, 86, 10, but no experimental results exist that allow quantifying the effective diffusivity of HDL. However, Karner et al. 44 give the diffusivities of ADP, albumin and LDL in blood plasma. The data in [44] is therefore normalized to the diffusivity of LDL in blood plasma and least-squares fitted to a rational function of the form $\frac{D_{\mathrm{Eff}, i}\left(R_{i}\right)}{D_{\mathrm{Eff}, \ell}}=\frac{e}{R_{i}^{f}}$. The least-squares fit yields $e=11.0 \mathrm{~nm}$ and $f=1.0$ such that

$$
D_{\mathrm{Eff}, i}\left(R_{i}\right)=\frac{11.0 \mathrm{~nm}}{R_{i}} D_{\mathrm{Eff}, \ell},
$$

which is plotted in Figure 10|(d). Here, the effective diffusion coefficients scales with the radius of particles and for HDL it yields $D_{\mathrm{Eff}, h}=2.33 D_{\mathrm{Eff}, \ell}=$ $2.94 \cdot 10^{-2} \frac{\mathrm{mm}^{2}}{\mathrm{~h}}$.

The rates of oxidative modification of HDL by macrophages $q_{h, m}$ and endothelial cells $q_{h, e}$ are crucial, but no experimental results exist that allows us to quantify them directly. Hence, the rates of HDL modification are calculated from the rates of LDL modification by considering the different structure and size of LDL and HDL. Therefore, we use

$$
q_{h, m}=\frac{q_{\ell, m}}{7.6} \frac{N_{\tilde{\ell}}}{N_{\tilde{h}}}, \quad q_{h, e}=\frac{q_{\ell, e}}{7.6} \frac{N_{\tilde{\ell}}}{N_{\tilde{h}}},
$$

where $N_{\tilde{\ell}}$ and $N_{\tilde{h}}$ correspond to the amount of lipid peroxide in modified LDL and HDL, respectively. The factor 7.6 represents the relative difference between the surface areas of LDL and HDL [16]. Using the values of $q_{\ell, m}, q_{\ell, e}, N_{\tilde{\ell}}$ and $N_{\tilde{h}}$ given in 92, Table 4 yields $q_{h, m}=1.64 \cdot 10^{-4} \frac{\mathrm{mm}^{3}}{\mathrm{~h}}$ and $q_{h, e}=6.21 \cdot 10^{-2} \frac{\mathrm{mm}}{\mathrm{h}}$.

Appendix A.4 Tranmural flow

The Darcy permeability $K$ must be estimated to fit to the murine physiology, i.e., to the murine intima-media thickness. The Darcy permeability is approximated by solving the norm of Eq. (9) for $K$ :

$$
K=\frac{\phi \mu\|\boldsymbol{u}\|}{\left\|\nabla p_{\mathrm{Med}}\right\|},
$$

where $\|\boldsymbol{u}\|$ and $\left\|\nabla p_{\text {Med }}\right\|$ correspond to the transmural filtration velocity and pressure gradient in the intima and media, respectively. The latter is approximated by the luminal blood pressure $\eta_{p}=100 \mathrm{mmHg}$ [1,69, 98, the pressure drop across the endothelium $\Delta p_{\text {End }}=18 \mathrm{mmHg}$ [89] and the adventitial pressure $p_{\mathrm{Adv}}=30 \mathrm{mmHg}[1,69,103]$ by: $\left\|\nabla p_{\mathrm{Med}}\right\|=\frac{\eta_{p}-\Delta p_{\mathrm{End}}-p_{\mathrm{Adv}}}{H}=\frac{52 \mathrm{mmHg}}{0.04 \mathrm{~mm}}$ $=1.73 \cdot 10^{5} \frac{\mathrm{Pa}}{\mathrm{mm}}$. Assuming a transmural filtration velocity $\|\boldsymbol{u}\|=6.41 \cdot 10^{-2} \frac{\mathrm{mm}}{\mathrm{h}}[29,103,63$, a porosity $\phi=0.15$ [1,74] and a dynamic viscosity of blood plasma $\mu=2.0 \cdot 10^{-7} \mathrm{~Pa} \mathrm{~h}$ [74,103] yields a Darcy permeability of $K=1.11 \cdot 10^{-14} \mathrm{~mm}^{2}$.

An overview of all parameters estimated and fitted so far is given in Table 2 
Appendix A.5 Wall Shear Stress-Dependent Parameters

There is experimental data by Sill et al. 81 that quantifies the hydraulic conductivity with respect to the WSS $\|\boldsymbol{\tau}\|$. Sun et al. 85] used these experimental results to parameterize the CSF (cf. Eq. (7)) which leads to: $\gamma_{p}=1.31 \cdot 10^{-1}$, $\mu_{p}=1.24 \cdot 10^{3} \frac{1}{\mathrm{~Pa}}$ and $\xi_{p}=1.86 \cdot 10^{1}$. In [85], the parameter $\mu_{p}$ was scaled so that it fits to a reference WSS value of $\|\bar{\tau}\|=1.68 \mathrm{~Pa}$. Even though the distribution of the reference WSS value $\|\overline{\boldsymbol{\tau}}\|$ within an animal is more or less uniform [79], it varies significantly in between different animal models [14. Therefore, the parameter $\mu_{p}$ of the CSF must be adapted to match the physiology of the animal under consideration, i.e., the murine physiology.

The same holds for the parameters $\xi_{\tau}$ and $\gamma_{\tau}$ of the macrophage recruitment submodel (cf. Eq. (3)) and the PSF (cf Eq. (6)). The estimated value $\xi_{\tau}=$ $1.95 \cdot 10^{-1} \mathrm{~Pa}$ corresponds to the human physiology with a reference WSS value $\|\bar{\tau}\|=1.16 \mathrm{~Pa}$ [14]. The parameter $\gamma_{\tau}$ is linked to a given reference WSS value $\|\overline{\boldsymbol{\tau}}\|$ by $\gamma_{\tau}=\frac{1}{30}\|\overline{\boldsymbol{\tau}}\|$, cf. 93]. A tabular overview of values for $\|\overline{\boldsymbol{\tau}}\|, \xi_{\tau}, \gamma_{\tau}$ and $\mu_{p}$ for different animal models and the normalized case is given in Table 3

\section{Appendix A.6 Plaque-Specific Parameters}

It remains to specify the concentrations of native LDL $\eta_{\ell}$ and HDL $\eta_{h}$ in blood and the WSS $\|\boldsymbol{\tau}\|$ that the model plaque is exposed to in vivo. These parameters characterize the physiology and diet of the plaque's host and the position of the plaque in the cardiovascular system. Thus, they cannot be set at fixed values, but their full ranges must be considered. The WSS $\|\boldsymbol{\tau}\|$ within a murine aortic arch is below approximately $7.7\|\bar{\tau}\|[93$. In a clinical context, the concentrations of LDL and HDL are measured by determining their cholesterol contents in blood. In humans, the physiological ranges lie approximately in between concentrations of $50-250 \frac{\mathrm{mg}}{\mathrm{dl}}$ of LDL blood cholesterol and $20-80 \frac{\mathrm{mg}}{\mathrm{dl}}$ of HDL blood cholesterol 68, which can be transformed into SI units of particles per volume using $\rho_{8}=4.36 \cdot 10^{17} \frac{1}{\mathrm{~g}}$ and $\rho_{13}=7.34 \cdot 10^{18} \frac{1}{\mathrm{~g}}$ (see [92], Table 1), respectively. In the case of mice with genetic modifications and highfat diets, the LDL and HDL cholesterol concentrations can rise above $3000 \frac{\mathrm{mg}}{\mathrm{dl}}$ and $400 \frac{\mathrm{mg}}{\mathrm{dl}}$, respectively [96,52. In general, however, mice show lower total blood cholesterol concentrations compared to humans, and in mice, as opposed to humans, HDL cholesterol is the predominant lipoprotein [52]. An overview of the full spectrum of LDL and HDL blood cholesterol concentrations as well as WSS is given in Table 4 .

\section{Appendix B Non-spatial Model}

A non-spatial version of the spatially resolved model developed in Section 2.2 is first given in this section. It is important to note that the non-spatial model is equivalent to the spatially resolved model, where the concentrations are 
homogeneous, e.g., induced by high effective diffusion coefficients $D_{\mathrm{Eff}, i} \rightarrow \infty$ $(i=\ell, \ldots, m)$. Subsequently, the non-spatial model is used to effectively perform sensitivity and stability analyses.

Appendix B.1 Model Overview and Proposition 1

The non-spatial model of early atherosclerosis consists of the parameterized ODE model developed in [92 enriched by the submodels of macrophage recruitment and lipoprotein fluxes in Sections 2.1.2 and 2.1.3. Altogether, the non-spatial model reads

$$
\begin{aligned}
\frac{\mathrm{d}}{\mathrm{d} t} \ell(t)= & -\mu_{\ell} \frac{\ell^{n_{\ell}}}{\left(\xi_{\ell}\right)^{n_{\ell}}+\ell^{n_{\ell}}} m-\left(q_{\ell, m} \ell m+\frac{q_{\ell, e}}{H} \ell\right) \cdot \frac{\left(k_{h}\right)^{n_{h}}}{\left(k_{h}\right)^{n_{h}}+h^{n_{h}}}+\frac{r_{\ell}(\ell,\|\boldsymbol{\tau}\|)}{H}, \\
\frac{\mathrm{d}}{\mathrm{d} t} \tilde{\ell}(t)= & -\mu_{\tilde{\ell}} \frac{\tilde{\ell}^{n_{\tilde{\ell}}}}{\left(\xi_{\tilde{\ell}}\right)^{n_{\tilde{\ell}}}+\tilde{\ell}_{\tilde{\ell}}} m+\left(q_{\ell, m} \ell m+\frac{q_{\ell, e}}{H} \ell\right) \cdot \frac{\left(k_{h}\right)^{n_{h}}}{\left(k_{h}\right)^{n_{h}}+h^{n_{h}}}+\frac{r_{\tilde{\ell}}(\tilde{\ell},\|\boldsymbol{\tau}\|)}{H}, \\
\frac{\mathrm{d}}{\mathrm{d} t} h(t)= & -q_{h, m} h m-\frac{q_{h, e}}{H} h+\frac{r_{h}(h,\|\boldsymbol{\tau}\|)}{H}, \\
\frac{\mathrm{d}}{\mathrm{d} t} f(t)= & +N_{f} \mu_{\ell} \frac{\ell^{n_{\ell}}}{\left(\xi_{\ell}\right)^{n_{\ell}}+\ell^{n_{\ell}}} m+N_{f} \mu_{\tilde{\ell}} \frac{\tilde{\ell}^{n_{\tilde{\ell}}}}{\left(\xi_{\tilde{\ell}}\right)^{n_{\tilde{\ell}}}+\tilde{\ell}_{\tilde{\ell}}^{n_{\tilde{\ell}}}} m-k_{f} \frac{\left(f-f_{\mathrm{Min}} m\right)^{2}}{f_{\mathrm{Max}} m-f} \\
& +k_{b} b-\mu_{f} \frac{h^{n_{f}}}{\left(\xi_{f}\right)^{n_{f}}+h^{n_{f}}}\left(f-f_{\mathrm{Min}} m\right)+\frac{r_{m}(\tilde{\ell},\|\boldsymbol{\tau}\|)}{H} f_{\mathrm{In}}, \\
\frac{\mathrm{d}}{\mathrm{d} t} b(t)= & +k_{f} \frac{\left(f-f_{\mathrm{Min}} m\right)^{2}}{f_{\mathrm{Max}} m-f} k_{b} b, \\
\frac{\mathrm{d}}{\mathrm{d} t} m(t)= & -\mu_{m} \frac{f^{n_{m}}}{\left(\xi_{m} m\right)^{n_{m}}+f^{n_{m}}} m+\frac{r_{m}(\tilde{\ell},\|\boldsymbol{\tau}\|)}{H},
\end{aligned}
$$

where the originally fixed-valued parameters corresponding to the fluxes of native LDL $r_{\ell}$, modified LDL $r_{\tilde{\ell}}$ and native HDL $r_{h}$ as well as the recruitment of macrophages $r_{m}$ were already notated including their explicit dependencies 
given by the submodels:

$$
\begin{aligned}
r_{\ell}(\ell,\|\boldsymbol{\tau}\|)= & P_{\ell} s_{\mathrm{P}}(\|\boldsymbol{\tau}\|)\left(\eta_{\ell}-\ell\right) \\
& +\left(1-\sigma_{\mathrm{F}, \ell}\right)\left(\omega_{\ell} \eta_{\ell}+\left(1-\omega_{\ell}\right) \ell\right) J_{\mathrm{Vol}}(p,\|\boldsymbol{\tau}\|), \\
r_{\tilde{\ell}}(\tilde{\ell},\|\boldsymbol{\tau}\|)= & -P_{\tilde{\ell}} s_{\mathrm{P}}(\|\boldsymbol{\tau}\|) \tilde{\ell}+\left(1-\sigma_{\mathrm{F}, \tilde{\ell}}\right)\left(1-\omega_{\tilde{\ell}} \tilde{\ell} J_{\mathrm{Vol}}(p,\|\boldsymbol{\tau}\|),\right. \\
r_{h}(h,\|\boldsymbol{\tau}\|)= & P_{h} s_{\mathrm{P}}(\|\boldsymbol{\tau}\|)\left(\eta_{h}-h\right) \\
& +\left(1-\sigma_{\mathrm{F}, h}\right)\left(\omega_{h} \eta_{h}+\left(1-\omega_{h}\right) h\right) J_{\mathrm{Vol}}(p,\|\boldsymbol{\tau}\|), \\
r_{m}(\tilde{\ell},\|\boldsymbol{\tau}\|)= & P_{m}\left(1-\delta_{m} \frac{k_{m}}{k_{m}+\tilde{\ell}}\right) \frac{\left(\xi_{\tau}\right)^{\nu_{\tau}}}{\left(\xi_{\tau}\right)^{\nu_{\tau}}+\|\boldsymbol{\tau}\|^{\nu_{\tau}}}, \\
J_{\mathrm{Vol}}(p,\|\boldsymbol{\tau}\|)= & L_{p} s_{\mathrm{L}}(\|\boldsymbol{\tau}\|)\left(\eta_{p}-p\right), \\
s_{\mathrm{P}}(\|\boldsymbol{\tau}\|)= & \frac{1}{\ln (2)} \ln \left(1+\zeta_{\tau} \frac{\gamma_{\tau}}{\|\boldsymbol{\tau}\|+\gamma_{\tau}}\right), \\
s_{\mathrm{L}}(\|\boldsymbol{\tau}\|)= & \gamma_{p} \ln \left(\mu_{p}\|\boldsymbol{\tau}\|+\xi_{p}\right) .
\end{aligned}
$$

The Initial conditions of the non-spatial model read analog to 92

$$
\begin{aligned}
& \ell(0)=0, \quad \tilde{\ell}(0)=\tilde{\ell}_{0}, \quad h(0)=0, \quad f(0)=f_{0} m_{0}, \\
& b(0)=b_{0} m_{0}, \quad m(0)=m_{0},
\end{aligned}
$$

where $m_{0}$ is adapted to due to the employed submodel of macrophage recruitment:

$$
\begin{aligned}
m_{0} & =\frac{r_{m}\left(\tilde{\ell}_{0},\|\boldsymbol{\tau}\|\right)}{H} \frac{\left(\xi_{m}\right)^{n_{m}}+\left(f_{0}\right)^{n_{m}}}{\mu_{m}\left(f_{0}\right)^{n_{m}}}, \\
b_{0} & =\frac{k_{f}}{k_{b}} \frac{\left(f_{0}-f_{\text {Min }}\right)^{2}}{f_{\text {Max }}-f_{0}} .
\end{aligned}
$$

Proposition 1. Let $\ell(t), \tilde{\ell}(t), h(t), f(t), b(t), m(t)(t \geq 0)$ be the unique and smooth solution of the initial value problem defined by Eqs. 30 - 33 with strictly positive parameters fulfilling $\left(1-\sigma_{\mathrm{F}, i}\right)\left(1-\omega_{i}\right) J_{\mathrm{Vol}}(p,\|\boldsymbol{\tau}\|) \leq P_{i} s_{\mathrm{P}}(\|\boldsymbol{\tau}\|)$, $0 \leq \omega_{i} \leq 1,0 \leq \sigma_{\mathrm{F}, i} \leq 1(i=\ell, \tilde{\ell}, h), 0 \leq p \leq \eta_{p}, f_{\mathrm{Min}} \leq f_{\mathrm{In}}<f_{\mathrm{Max}}$ and $f_{\text {Min }} \leq f_{0}<f_{\text {Max }}$. Then the solution satisfies:

1. $\ell(t), \tilde{\ell}(t), h(t), f(t), b(t), m(t) \geq 0$ for all $t \geq 0$.

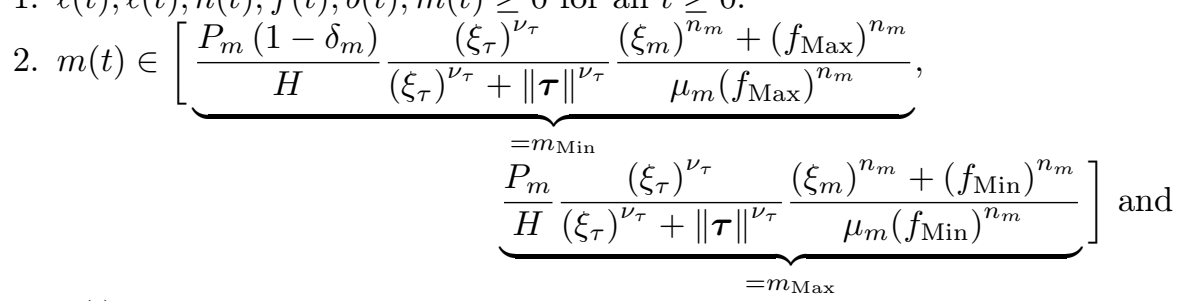

$\frac{f(t)}{m(t)} \in\left[f_{\mathrm{Min}}, f_{\mathrm{Max}}\right]$ for all $t \geq 0$.

3. $\ell(t), \tilde{\ell}(t), h(t), f(t), m(t)$ are bounded. 
Proof. The proof of the Proposition 1 is similar to the proof of the Proposition given in [92]. First, it follows from $\eta_{p} \geq p$ that

$$
J_{\mathrm{Vol}}(p,\|\boldsymbol{\tau}\|) \stackrel{\sqrt[31]{=}}{=} L_{p} s_{\mathrm{L}}(\|\boldsymbol{\tau}\|)\left(\eta_{p}-p\right) \geq 0
$$

To prove the positivity of $\ell$, i.e., $\ell(t) \geq 0 \forall t \geq 0$ it is sufficient to note that $\ell(0)=0$ and that $\ell(t)=0$ implies

$$
\frac{\mathrm{d}}{\mathrm{d} t} \ell(t) \stackrel{\text { 30 }}{=} \frac{P_{\ell} s_{\mathrm{P}}(\|\boldsymbol{\tau}\|) \eta_{\ell}+\left(1-\sigma_{\mathrm{F}, \ell}\right) \omega_{\ell} \eta_{\ell} J_{\mathrm{Vol}}(p,\|\boldsymbol{\tau}\|)}{H} \stackrel{34,1 \geq \sigma_{\mathrm{F}, \ell}}{>} 0
$$

due to the strict positivity of the parameters. The positivities of $h, m$ and $\tilde{\ell}$ are proved in an analog fashion. Since $\frac{f(0)}{m(0)}=f_{0} \geq f_{\text {Min }}$ and $\frac{f(t)}{m(t)}=f_{\text {Min }}$ implies

$$
\begin{aligned}
\frac{\mathrm{d}}{\mathrm{d} t}\left(\frac{f(t)}{m(t)}\right) & =\frac{\frac{\mathrm{d}}{\mathrm{d} t} f(t)-\frac{f(t)}{m(t)} \frac{\mathrm{d}}{\mathrm{d} t} m(t)}{m(t)} \\
& \geq \frac{\frac{r_{m}(\tilde{\ell}(t),\|\boldsymbol{\tau}\|)}{H} f_{\operatorname{In}}-f_{\operatorname{Min}} \frac{r_{m}(\tilde{\ell}(t),\|\boldsymbol{\tau}\|)}{H}}{m(t)} \geq 0,
\end{aligned}
$$

it holds $\frac{f(t)}{m(t)} \geq f_{\text {Min }} \forall t \geq 0$. This also implies the positivity of $f$. Given that $\frac{f(0)}{m(0)}=f_{0}<f_{\text {Max }}$ and that $\frac{f(t)}{m(t)} \rightarrow f_{\text {Max }}$ implies

$$
\frac{\mathrm{d}}{\mathrm{d} t}\left(\frac{f(t)}{m(t)}\right)=\frac{\frac{\mathrm{d}}{\mathrm{d} t} f(t)-\frac{f(t)}{m(t)} \frac{\mathrm{d}}{\mathrm{d} t} m(t)}{m(t)} \longrightarrow-\infty
$$

it follows $\frac{f(t)}{m(t)} \leq f_{\text {Max }} \forall t \geq 0$. Hence, it is concluded that

$$
\frac{f(t)}{m(t)} \in\left[f_{\mathrm{Min}}, f_{\mathrm{Max}}\right] \forall t \geq 0
$$

The positivity of $b$ follows since $b(0)=0$ and $b(t)=0$ implies

$$
\frac{\mathrm{d}}{\mathrm{d} t} b(t) \stackrel{30}{=} k_{f} \frac{\left(f(t)-f_{\text {Min }} m\right)^{2}}{f_{\text {Max }} m-f(t)} \stackrel{[38)}{\geq} 0
$$

which finishes the proof of 1 . (It also follows that the time-dependent solution $(\ell(t), \tilde{\ell}(t), h(t), f(t), b(t), m(t)), t \geq 0$ of the initial value problem is unique and smooth because the smoothness of the right-hand side of the ODE (30) is now straightforward to show.)

Using 38

$$
\begin{aligned}
\frac{\mathrm{d}}{\mathrm{d} t} m(t) & \stackrel{\text { 30, } 38}{\geq}-\underbrace{\mu_{m} \frac{\left(f_{\mathrm{Max}}\right)^{n_{m}}}{\left(\xi_{m}\right)^{n_{m}}+\left(f_{\mathrm{Max}}\right)^{n_{m}}}}_{=: z_{\mathrm{Max}}} m+\frac{r_{m}(\tilde{\ell}(t),\|\boldsymbol{\tau}\|)}{H} \\
& \geq-z_{\operatorname{Max}} m+\frac{r_{m}(0,\|\boldsymbol{\tau}\|)}{H}
\end{aligned}
$$


holds, and by solving this ordinary differential inequality with associated initial condition $m(0)=m_{0}$ it follows

$$
\begin{aligned}
m(t) & \stackrel{40}{\geq} m_{0} \exp \left(-z_{\mathrm{Max}} t\right)+\frac{r_{m}(0,\|\boldsymbol{\tau}\|)}{H z_{\mathrm{Max}}}\left(1-\exp \left(-z_{\mathrm{Max}} t\right)\right) \\
& \geq \min \left(m_{0}, \frac{r_{m}(0,\|\boldsymbol{\tau}\|)}{H z_{\mathrm{Max}}}\right) \stackrel{\sqrt[33]{,}, f_{\text {Max }}>f_{0} \frac{r_{m}(0,\|\boldsymbol{\tau}\|)}{H z_{\operatorname{Max}}}}{=} \\
& =\frac{r_{m}(0,\|\boldsymbol{\tau}\|)}{H} \frac{\left(\xi_{m}\right)^{n_{m}}+\left(f_{\mathrm{Max}}\right)^{n_{m}}}{\mu_{m}\left(f_{\mathrm{Max}}\right)^{n_{m}}} \forall t \geq 0 .
\end{aligned}
$$

In an analog manner, the upper bound for $m(t)$ is found, leading to

$$
\begin{aligned}
m(t) \in[\underbrace{\frac{r_{m}(0,\|\boldsymbol{\tau}\|)}{H} \frac{\left(\xi_{m}\right)^{n_{m}}+\left(f_{\mathrm{Max}}\right)^{n_{m}}}{\mu_{m}\left(f_{\mathrm{Max}}\right)^{n_{m}}}}_{=: m_{\text {Min }}} \\
, \underbrace{\frac{r_{m}(\infty,\|\boldsymbol{\tau}\|)}{H} \frac{\left(\xi_{m}\right)^{n_{m}}+\left(f_{\mathrm{Min}}\right)^{n_{m}}}{\mu_{m}\left(f_{\mathrm{Min}}\right)^{n_{m}}}}_{=: m_{\operatorname{Max}}}] \forall t \geq 0
\end{aligned}
$$

which finishes the proof of 2 .

Due to $\left(1-\sigma_{\mathrm{F}, \ell}\right)\left(1-\omega_{\ell}\right) J_{\mathrm{Vol}}(p,\|\boldsymbol{\tau}\|) \leq P_{\ell} s_{\mathrm{P}}(\|\boldsymbol{\tau}\|)$, the boundedness of $\ell(t)$ is given by

$$
\begin{aligned}
\frac{\mathrm{d}}{\mathrm{d} t} \ell(t) & \stackrel{300, \sqrt[322]{\leq}-\left(q_{\ell, m} m_{\mathrm{Min}}+\frac{q_{\ell, e}}{H}\right) \ell(t)+\frac{r_{\ell}(\ell,\|\boldsymbol{\tau}\|)}{H}}{=}-\left(q_{\ell, m} m_{\mathrm{Min}}+\frac{q_{\ell, e}}{H}\right) \ell(t)+\left(P_{\ell} s_{\mathrm{P}}(\|\boldsymbol{\tau}\|)+\left(1-\sigma_{\mathrm{F}, \ell}\right) \omega_{\ell} J_{\mathrm{Vol}}(p,\|\boldsymbol{\tau}\|)\right) \frac{\eta_{\ell}}{H} \\
& +\underbrace{\left(-P_{\ell} s_{\mathrm{P}}(\|\boldsymbol{\tau}\|)+\left(1-\sigma_{\mathrm{F}, \ell}\right)\left(1-\omega_{\ell}\right) J_{\mathrm{Vol}}(p,\|\boldsymbol{\tau}\|)\right)}_{\leq 0} \frac{\ell(t)}{H} \\
\leq & -\left(q_{\ell, m} m_{\mathrm{Min}}+\frac{q_{\ell, e}}{H}\right) \ell(t)+\left(P_{\ell} s_{\mathrm{P}}(\|\boldsymbol{\tau}\|)+\left(1-\sigma_{\mathrm{F}, \ell}\right) \omega_{\ell} J_{\mathrm{Vol}}(p,\|\boldsymbol{\tau}\|)\right) \frac{\eta_{\ell}}{H}
\end{aligned}
$$

since the solution of the ordinary differential inequality (with associated initial condition $\ell(0)=0$ ) is bounded by

$$
\begin{aligned}
\ell(t) \stackrel{43}{\leq} \frac{\left(P_{\ell} s_{\mathrm{P}}(\|\boldsymbol{\tau}\|)+\left(1-\sigma_{\mathrm{F}, \ell}\right) \omega_{\ell} J_{\mathrm{Vol}}(p,\|\boldsymbol{\tau}\|)\right) \eta_{\ell}}{H q_{\ell, m} m_{\mathrm{Min}}+q_{\ell, e}} \\
\left(1-\exp \left(-\left(q_{\ell, m} m_{\mathrm{Min}}+\frac{q_{\ell, e}}{H}\right) t\right)\right) \\
\quad \leq \frac{\left(P_{\ell} s_{\mathrm{P}}(\|\boldsymbol{\tau}\|)+\left(1-\sigma_{\mathrm{F}, \ell}\right) \omega_{\ell} J_{\mathrm{Vol}}(p,\|\boldsymbol{\tau}\|)\right) \eta_{\ell}}{H q_{\ell, m} m_{\mathrm{Min}}+q_{\ell, e}}<\infty .
\end{aligned}
$$

In an analog manner, the boundedness of $\tilde{\ell}(t)$ and $h(t)$ are proved which finishes the proof of Proposition 1. 
Appendix B.2 Sensitivity Analysis

The parameters estimated in Appendix A have a degree of uncertainty due to their origins, indifferent pieces of research used as experimental models, and measurement errors. To quantify the effect of uncertainties in the parameters on the computational results of the model, a local sensitivity analysis using the method of a metabolic control analysis [105] is performed. However, the computational cost to do this analysis for the spatially resolved model is too large, so that a metabolic control analysis using the spatially resolved model is not achievable. Therefore, we use the non-spatial model from Appendix B.1 with prescribed subendothelial pressure $p=\eta_{p}-\Delta p_{\text {End }}=82 \mathrm{mmHg}=$ $1.09 \cdot 10^{4} \mathrm{~Pa}$ [89, 94, 69, 15], LDL cholesterol concentration $\eta_{\ell}=150 \frac{\mathrm{mg}}{\mathrm{dl}}, \mathrm{HDL}$ cholesterol concentration $\eta_{h}=50 \frac{\mathrm{mg}}{\mathrm{dl}}$ and WSS $\|\boldsymbol{\tau}\|=10 \%\|\overline{\boldsymbol{\tau}}\|$.

The metabolic control analysis is performed in the same way as in [105, 92]. Accordingly, we compare the normalized partial derivatives of all concentrations with respect to all non-spatial parameters $p$ given in Table 2 at time $T=$ 100 weeks. The uncertainties of the remaining non-spatial parameters were previously addressed in [92]. As proposed in [105], the partial derivatives are estimated by using forward finite difference approximations with a sufficiently small variation parameter $\varepsilon=0.1 \%$. Hence, the metabolic control coefficient $\operatorname{MCC}(\ell, p)$ of LDL $\ell$ with respect to a model parameter $p$ is computed by

$$
\operatorname{MCC}(\ell, p)=\frac{1}{\ell_{p}(T)} \frac{\partial}{\partial p} \ell_{p}(T) \approx \frac{1}{\ell_{p}(T)} \frac{\ell_{p+\varepsilon \%}(T)-\ell_{p}(T)}{\varepsilon},
$$

where $\ell_{p+\varepsilon} \%$ denotes the concentration of native LDL $\ell$ computed with the parameter $p$ perturbed by $\varepsilon$ percent. The metabolic control coefficients of all other species are computed in the same manner. The metabolic control coefficients of all species of the non-spatial model with respect to all non-spatial parameters from Table 2 are plotted in Figure 11.

From the sensitivity analysis it follows that variations of the filtration reflection coefficients of LDL $\sigma_{\mathrm{F}, \ell}$ and $\mathrm{HDL} \sigma_{\mathrm{F}, h}$ have the largest influence on the computational results. While the impact of the filtration reflection coefficient of HDL $\sigma_{\mathrm{F}, h}$ is still moderate, the effect of slight deviation of $\sigma_{\mathrm{F}, \ell}$ is severe. Even though the literature in general agrees on $\sigma_{\mathrm{F}, \ell} \approx 9.97 \cdot 10^{-1}$,44, $103,86,74,1$, both parameters $\sigma_{\mathrm{F}, \ell}$ and $\sigma_{\mathrm{F}, h}$ require further attention, especially by experimental communities.

\section{Appendix B.3 Stability Analysis}

The outcome of the spatially resolved model is strongly dependent on blood cholesterol concentrations of LDL $\eta_{\ell}$ and HDL $\eta_{h}$ and the WSS $\|\boldsymbol{\tau}\|$ which characterize the physiology and diet of the host and the position of the individual plaque within the cardiovascular system. They can - depending on the species of host, its predisposition and its diet - vary by several orders of magnitude (cf. Table 4) which results in qualitatively different predicted long-term outcomes. 

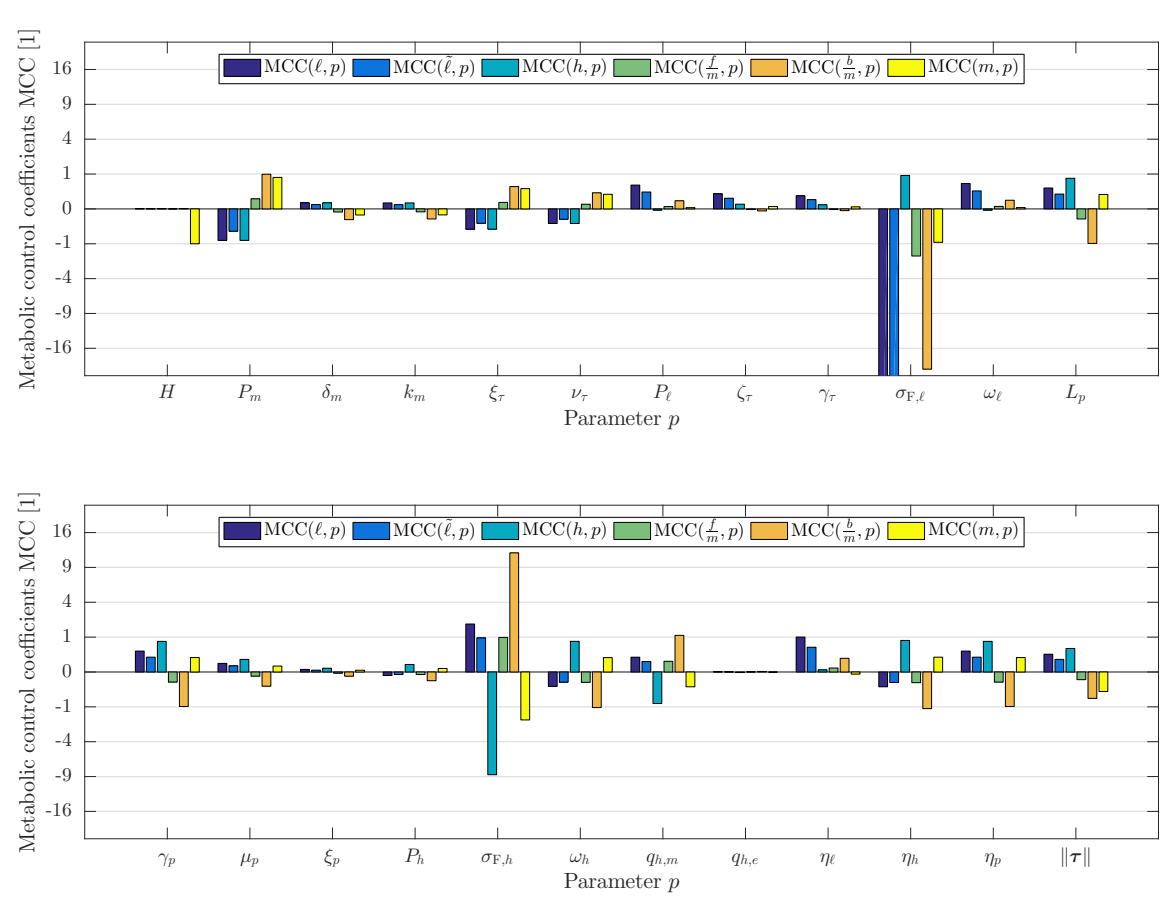

Fig. 11 Sensitivity analysis of the non-spatial model. Metabolic control coefficients of native LDL $\ell$, modified LDL $\tilde{\ell}$, HDL $h$, intracellular free cholesterol per macrophage $\frac{f}{m}$, intracellular cholesterol ester per macrophage $\frac{b}{m}$ and macrophages $m$ with respect to all non-spatial parameters $p$ in Table 2. The values of the truncated bars are $\operatorname{MCC}\left(\ell, \sigma_{\mathrm{F}, \ell}\right)=-175.90$ and $\operatorname{MCC}\left(\tilde{\ell}, \sigma_{\mathrm{F}, \ell}\right)=-92.68$.

Due to the high computational cost of the spatially resolved model, a stability analysis is performed using the non-spatial model from Appendix B.1.

Proposition 1 from Appendix B.1 applies, as all its requirements are fulfilled by the non-spatial model with the complete parameter set from Tables 2 and 3 and [92, Table 4. Hence, the steady-state concentrations of macrophages $\widehat{m}$ and intracellular free cholesterol per macrophage $\frac{\widehat{f}}{\widehat{m}}$ satisfy

$$
\begin{aligned}
& \widehat{m} \in\left[m_{\mathrm{Min}}, m_{\mathrm{Max}}\right]=\left[0.04 \cdot 10^{8}, 14.96 \cdot 10^{8}\right] \frac{1}{\mathrm{~mm}^{3}}, \\
& \frac{\widehat{f}}{\widehat{m}} \in\left[f_{\mathrm{Min}}, f_{\mathrm{Max}}\right]=\left[1.22 \cdot 10^{10}, 7.15 \cdot 10^{10}\right]
\end{aligned}
$$

and only the concentration of cholesterol ester $b$ can be unbounded. The boundedness of intracellular cholesterol ester $b$, the steady-state concentration of intracellular free cholesterol per macrophages $\frac{\widehat{f}}{\hat{m}}$ and the steady-state density of macrophages $\widehat{m}$ are computed in the crucial ranges of $\eta_{\ell}, \eta_{h}$ and $\|\boldsymbol{\tau}\|$. High LDL cholesterol, low HDL cholesterol and low WSS promote atherosclerosis, 
and so the ranges that we use for the stability analysis are $\eta_{\ell} \in[10,800] \frac{\mathrm{mg}}{\mathrm{dl}}$, $\eta_{h} \in[20,100] \frac{\mathrm{mg}}{\mathrm{dl}}$ and $\|\boldsymbol{\tau}\| \in[5 \%, 40 \%]\|\overline{\boldsymbol{\tau}}\|$, cf. Table 4 . The points where analysis is done are on an equidistant grid of the specified parameter space. The numerical results for varying blood cholesterol concentrations $\eta_{\ell}$ and $\eta_{h}$ and four different WSS levels $\|\boldsymbol{\tau}\|$ are plotted in Figure 12 .

(a)

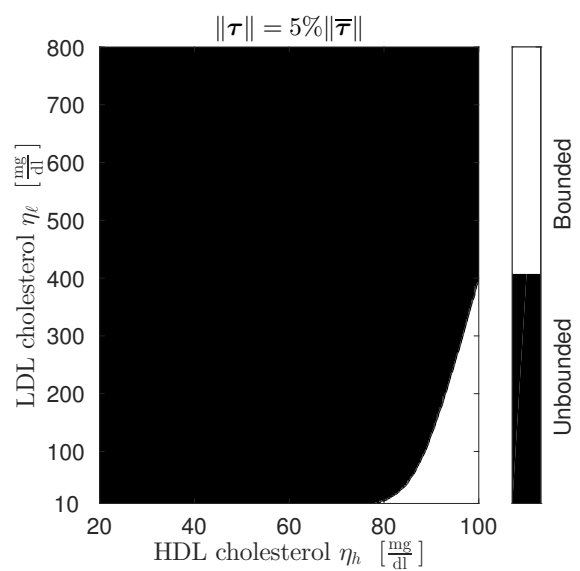

(c)

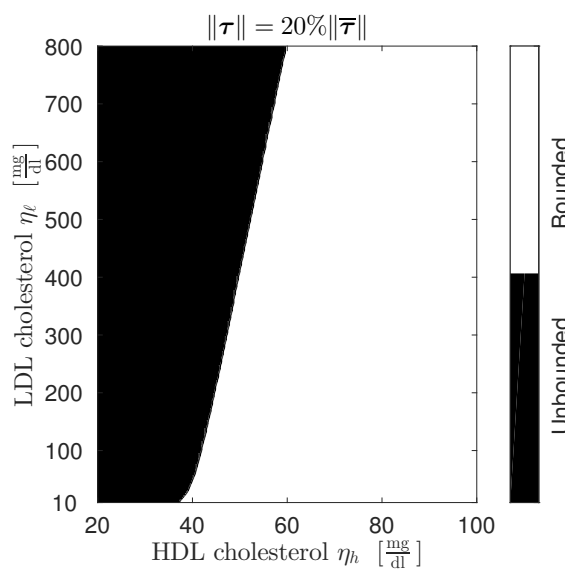

(b)

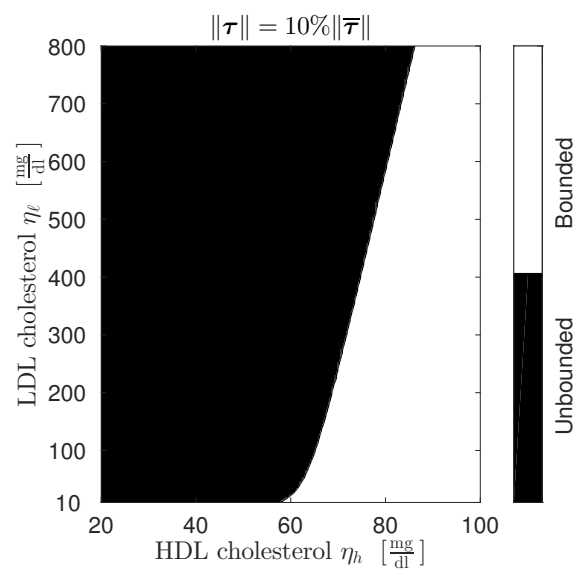

(d)

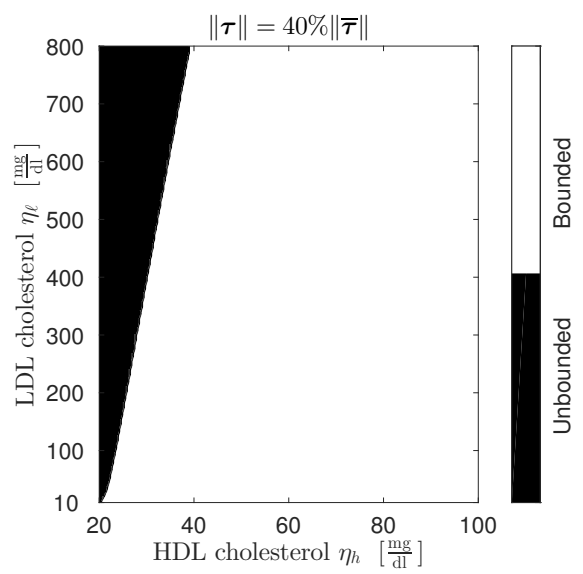

Fig. 12 Stability analysis of the non-spatial model. Boundedness of intracellular cholesterol ester $[(\mathrm{a})-(\mathrm{d})]$, steady-state concentration of intracellular free cholesterol per macrophage $\frac{\widehat{f}}{\widehat{m}} \sqrt{(\mathrm{e})}(\mathrm{h})$, and steady-state density of macrophages $\widehat{m}$ (i) (1) are predicted for varying LDL and HDL cholesterol concentrations $\eta_{\ell}$ and $\eta_{h}$ and various WSS $\|\boldsymbol{\tau}\|$. 
(e)

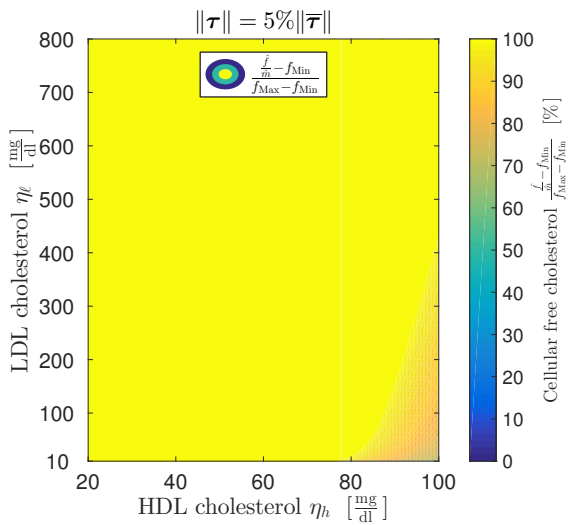

(g)

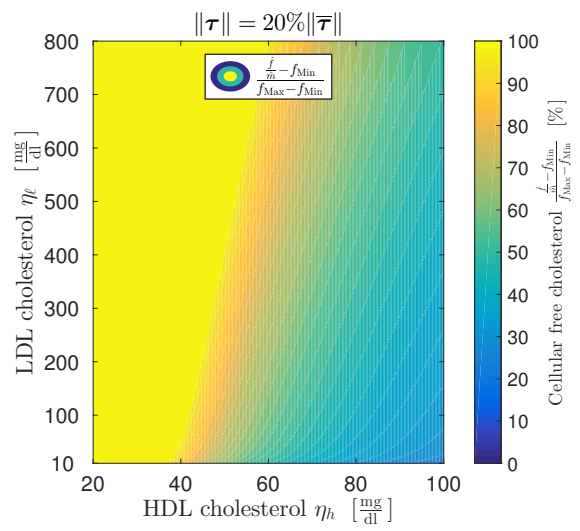

(f)

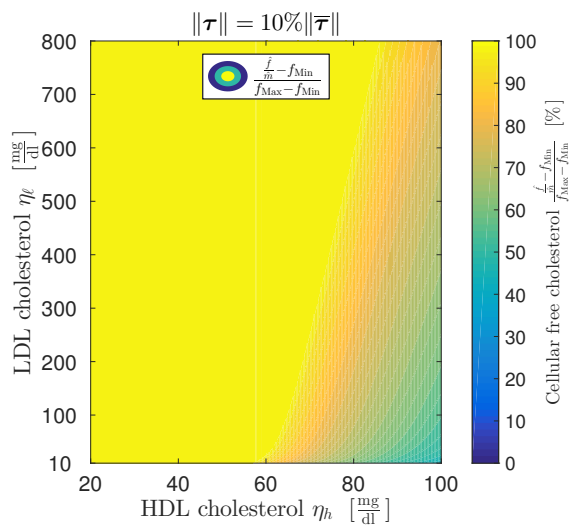

(h)

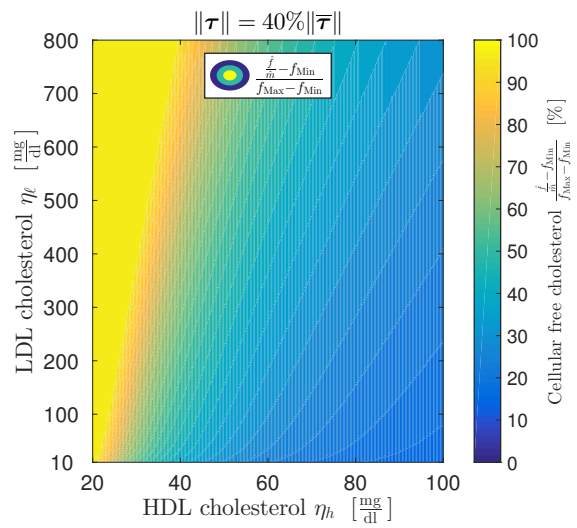

Fig. 12 Continued. 
(i)

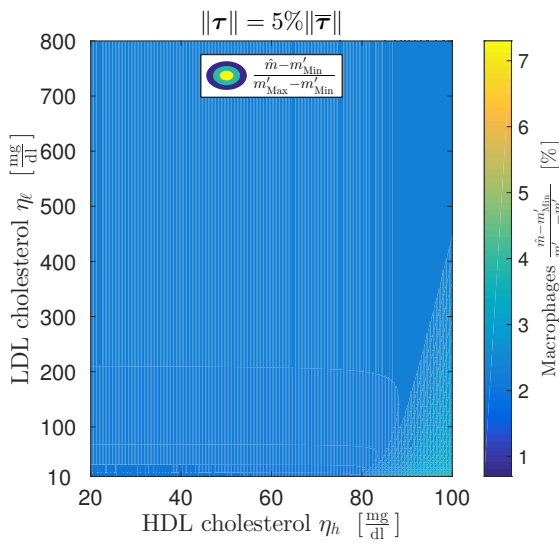

(k)

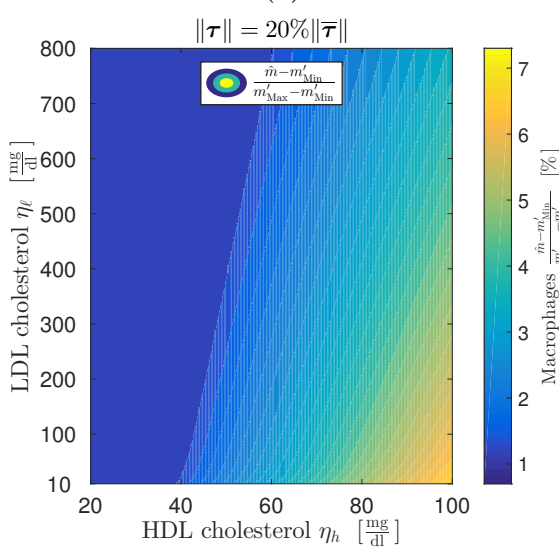

(j)

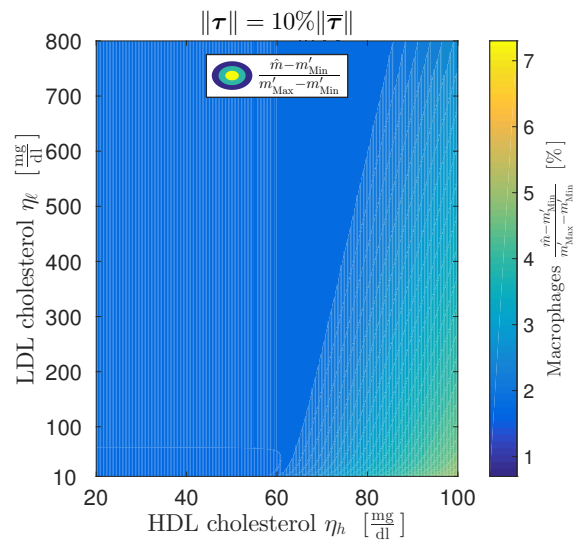

(1)

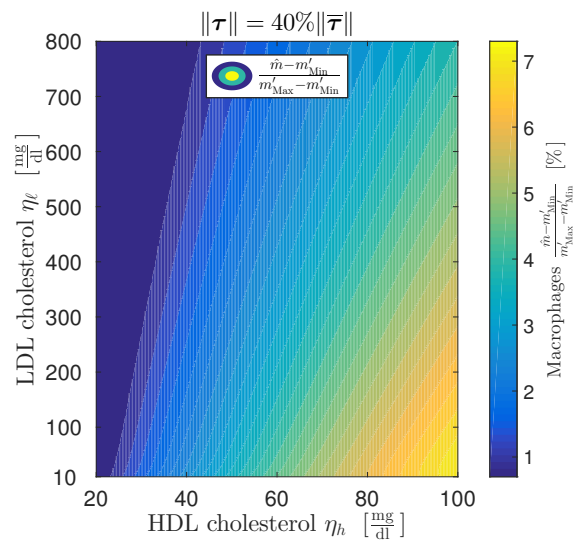

Fig. 12 Continued. 


\section{References}

1. Ai, L., Vafai, K.: A coupling model for macromolecule transport in a stenosed arterial wall. International Journal of Heat and Mass Transfer 49(9), 1568-1591 (2006)

2. Alon, R., Kassner, P.D., Carr, M.W., Finger, E.B., Hemler, M.E., Springer, T.A.: The integrin VLA-4 supports tethering and rolling in flow on VCAM-1. The Journal of Cell Biology 128(6), 1243-1253 (1995)

3. Bear, J., Bachmat, Y.: Introduction to modeling of transport phenomena in porous media, vol. 4. Springer Science and Business Media (2012)

4. Berliner, J., Territo, M., Sevanian, A., Ramin, S., Kim, J.A., Bamshad, B., Esterson, M., Fogelman, A.M.: Minimally modified low density lipoprotein stimulates monocyte endothelial interactions. Journal of Clinical Investigation 85(4), 1260 (1990)

5. Bratzler, R.L., Chisolm, G.M., Colton, C.K., Smith, K.A., Lees, R.S.: The distribution of labeled low-density lipoproteins across the rabbit thoracic aorta in vivo. Atherosclerosis 28(3), 289-307 (1977)

6. Brown, A.J., Teng, Z., Evans, P.C., Gillard, J.H., Samady, H., Bennett, M.R.: Role of biomechanical forces in the natural history of coronary atherosclerosis. Nature Reviews Cardiology 13, $210-220$ (2016)

7. Brown, M.S., Goldstein, J.L., Krieger, M., Ho, Y., Anderson, R.: Reversible accumulation of cholesteryl esters in macrophages incubated with acetylated lipoproteins. The Journal of Cell Biology 82(3), 597-613 (1979)

8. Brown, M.S., Ho, Y., Goldstein, J.: The cholesteryl ester cycle in macrophage foam cells. Continual hydrolysis and re-esterification of cytoplasmic cholesteryl esters. Journal of Biological Chemistry 255(19), 9344-9352 (1980)

9. Bulelzai, M.A., Dubbeldam, J.L.: Long time evolution of atherosclerotic plaques. Journal of Theoretical Biology 297, 1-10 (2012)

10. Calvez, V., Houot, J.G., Meunier, N., Raoult, A., Rusnakova, G.: Mathematical and numerical modeling of early atherosclerotic lesions. ESAIM: Proceedings and Surveys 30, 1 - 14 (2010)

11. Chalmers, A.D., Bursill, C.A., Myerscough, M.R.: Nonlinear dynamics of early atherosclerotic plaque formation may determine the efficacy of high density lipoproteins (hdl) in plaque regression. PloS One 12(11), e0187,674 (2017)

12. Chalmers, A.D., Cohen, A., Bursill, C.A., Myerscough, M.R.: Bifurcation and dynamics in a mathematical model of early atherosclerosis. Journal of Mathematical Biology 71(6-7), 1451-1480 (2015)

13. Chang, Y.S., Yaccino, J.A., Lakshminarayanan, S., Frangos, J.A., Tarbell, J.M.: Shearinduced increase in hydraulic conductivity in endothelial cells is mediated by a nitric oxide-dependent mechanism. Arteriosclerosis, Thrombosis, and Vascular Biology 20(1), 35-42 (2000)

14. Cheng, C., Helderman, F., Tempel, D., Segers, D., Hierck, B., Poelmann, R., van Tol, A., Duncker, D.J., Robbers-Visser, D., Ursem, N.T., et al.: Large variations in absolute wall shear stress levels within one species and between species. Atherosclerosis 195(2), 225-235 (2007)

15. Cilla, M., Peña, E., Martínez, M.A.: Mathematical modelling of atheroma plaque formation and development in coronary arteries. Journal of The Royal Society Interface 11(90), 20130,866 (2014)

16. Cobbold, C., Sherratt, J., Maxwell, S.: Lipoprotein oxidation and its significance for atherosclerosis: a mathematical approach. Bulletin of Mathematical Biology 64(1), 65-95 (2002)

17. Cominacini, L., Garbin, U., Pasini, A.F., Davoli, A., Campagnola, M., Contessi, G.B., Pastorino, A.M., Cascio, V.L.: Antioxidants inhibit the expression of intercellular cell adhesion molecule- 1 and vascular cell adhesion molecule-1 induced by oxidized LDL on human umbilical vein endothelial cells. Free Radical Biology and Medicine 22(1), 117-127 (1997)

18. Coussy, O.: Poromechanics. John Wiley and Sons (2004)

19. Crosetto, P., Reymond, P., Deparis, S., Kontaxakis, D., Stergiopulos, N., Quarteroni, A.: Fluid-structure interaction simulation of aortic blood flow. Computers and Fluids 43(1), 46-57 (2011) 
20. Curmi, P.A., Juan, L., Tedgui, A.: Effect of transmural pressure on low density lipoprotein and albumin transport and distribution across the intact arterial wall. Circulation Research 66(6), 1692-1702 (1990)

21. Cybulsky, M.I., Gimbrone Jr, M.A.: Endothelial expression of a mononuclear leukocyte adhesion molecule during atherogenesis. Science 251(4995), 788 (1991)

22. Donea, J., Huerta, A.: Finite element methods for flow problems. John Wiley and Sons (2003)

23. El-Bassiouni, E., Helmy, M., El-Zoghby, S., Kamel, E.N., Hosny, R.: Relationship between level of circulating modified LDL and the extent of coronary artery disease in type 2 diabetic patients. British Journal of Biomedical Science 64(3), 109-116 (2007)

24. Faxon, D.P., Fuster, V., Libby, P., Beckman, J.A., Hiatt, W.R., Thompson, R.W., Topper, J.N., Annex, B.H., Rundback, J.H., Fabunmi, R.P., et al.: Atherosclerotic vascular disease conference writing group III: Pathophysiology. Circulation 109(21), 2617-2625 (2004)

25. Feintuch, A., Ruengsakulrach, P., Lin, A., Zhang, J., Zhou, Y.Q., Bishop, J., Davidson, L., Courtman, D., Foster, F.S., Steinman, D.A., et al.: Hemodynamics in the mouse aortic arch as assessed by MRI, ultrasound, and numerical modeling. American Journal of Physiology-Heart and Circulatory Physiology 292(2), H884-H892 (2007)

26. Feng, B., Yao, P.M., Li, Y., Devlin, C.M., Zhang, D., Harding, H.P., Sweeney, M., Rong, J.X., Kuriakose, G., Fisher, E.A., et al.: The endoplasmic reticulum is the site of cholesterol-induced cytotoxicity in macrophages. Nature Cell Biology 5(9), 781-792 (2003)

27. Figueroa, C.A., Baek, S., Taylor, C.A., Humphrey, J.D.: A computational framework for fluid-solid-growth modeling in cardiovascular simulations. Computer Methods in Applied Mechanics and Engineering 198(45-46), 3583-3602 (2009)

28. Fok, P.W.: Multi-layer mechanical model of Glagov remodeling in coronary arteries: Differences between in-vivo and ex-vivo measurements. PloS One 11(7), e0159,304 (2016)

29. Formaggia, L., Quarteroni, A., Veneziani, A.: Cardiovascular Mathematics: Modeling and simulation of the circulatory system, vol. 1. Springer (2010)

30. Friedman, A., Hao, W.: A mathematical model of atherosclerosis with reverse cholesterol transport and associated risk factors. Bulletin of Mathematical Biology 77(5), 758-781 (2015)

31. Frostegard, J., Haegerstrand, A., Gidlund, M., Nilsson, J.: Biologically modified LDL increases the adhesive properties of endothelial cells. Atherosclerosis 90(2-3), 119-126 (1991)

32. Gordon, S., Taylor, P.R.: Monocyte and macrophage heterogeneity. Nature Reviews Immunology 5(12), 953-964 (2005)

33. Hansson, G.K., Libby, P.: The immune response in atherosclerosis: a double-edged sword. Nature Reviews Immunology 6(7), 508-519 (2006)

34. Hao, W., Friedman, A.: The LDL-HDL profile determines the risk of atherosclerosis: a mathematical model. PloS One 9(3), e90,497 (2014)

35. Henriksen, T., Mahoney, E.M., Steinberg, D.: Enhanced macrophage degradation of biologically modified low density lipoprotein. Arteriosclerosis, Thrombosis, and Vascular Biology 3(2), 149-159 (1983)

36. Herrmann, R.A., Malinauskas, R.A., Truskey, G.A.: Characterization of sites with elevated LDL permeability at intercostal, celiac, and iliac branches of the normal rabbit aorta. Arteriosclerosis, Thrombosis, and Vascular Biology 14(2), 313-323 (1994)

37. Hoffman, A.H., Teng, Z., Zheng, J., Wu, Z., Woodard, P.K., Billiar, K.L., Wang, L., Tang, D.: Stiffness properties of adventitia, media, and full thickness human atherosclerotic carotid arteries in the axial and circumferential directions. Journal of Biomechanical Engineering 139(12), 124,501 (2017)

38. Holzapfel, G.A., Sommer, G., Gasser, C.T., Regitnig, P.: Determination of layer-specific mechanical properties of human coronary arteries with nonatherosclerotic intimal thickening and related constitutive modeling. American Journal of Physiology-Heart and Circulatory Physiology 289(5), H2048-H2058 (2005)

39. Hsiai, T.K., Cho, S.K., Wong, P.K., Ing, M., Salazar, A., Sevanian, A., Navab, M. Demer, L.L., Ho, C.M.: Monocyte recruitment to endothelial cells in response to oscillatory shear stress. The FASEB Journal 17(12), 1648-1657 (2003) 
40. Huang, Z., Tarbell, J.: Numerical simulation of mass transfer in porous media of blood vessel walls. American Journal of Physiology - Heart and Circulatory Physiology 273(1), H464-H477 (1997)

41. Huo, Y., Hafezi-Moghadam, A., Ley, K.: Role of vascular cell adhesion molecule1 and fibronectin connecting segment-1 in monocyte rolling and adhesion on early atherosclerotic lesions. Circulation Research 87(2), 153-159 (2000)

42. Jeng, J.R., Chang, C.H., Shih-Ming, S., Hui-Chong, C.: Oxidized low-density lipoprotein enhances monocyte-endothelial cell binding against shear-stress-induced detachment. Biochimica et Biophysica Acta (BBA)-Molecular Cell Research 1178(2), 221-227 (1993)

43. Karner, G., Perktold, K.: Effect of endothelial injury and increased blood pressure on albumin accumulation in the arterial wall: a numerical study. Journal of Biomechanics 33(6), 709-715 (2000)

44. Karner, G., Perktold, K., Zehentner, H.P.: Computational modeling of macromolecule transport in the arterial wall. Computer Methods in Biomechanics and Biomedical Engineering 4(6), 491-504 (2001)

45. Kedem, O., Katchalsky, A.: Thermodynamic analysis of the permeability of biological membranes to non-electrolytes. Biochimica et Biophysica Acta 27, 229-246 (1958)

46. Kontush, A., Chapman, M.J.: Functionally defective high-density lipoprotein: a new therapeutic target at the crossroads of dyslipidemia, inflammation, and atherosclerosis. Pharmacological Reviews 58(3), 342-374 (2006)

47. Koshiba, N., Ando, J., Chen, X., Hisada, T.: Multiphysics simulation of blood flow and LDL transport in a porohyperelastic arterial wall model. Journal of Biomechanical Engineering 129(3), 374-385 (2007)

48. Ku, D.N., Giddens, D.P., Zarins, C.K., Glagov, S.: Pulsatile flow and atherosclerosis in the human carotid bifurcation. positive correlation between plaque location and low oscillating shear stress. Arteriosclerosis, Thrombosis, and Vascular Biology 5(3), 293-302 (1985)

49. Kunjathoor, V.V., Febbraio, M., Podrez, E.A., Moore, K.J., Andersson, L., Koehn, S., Rhee, J.S., Silverstein, R., Hoff, H.F., Freeman, M.W.: Scavenger receptors class AI/II and CD36 are the principal receptors responsible for the uptake of modified low density lipoprotein leading to lipid loading in macrophages. Journal of Biological Chemistry $\mathbf{2 7 7}(51)$, 49,982-49,988 (2002)

50. Lasseux, D., Ahmadi, A., Arani, A.A.A.: Two-phase inertial flow in homogeneous porous media: a theoretical derivation of a macroscopic model. Transport in Porous Media 75(3), 371-400 (2008)

51. Leake, D.S., Rankin, S.M.: The oxidative modification of low-density lipoproteins by macrophages. Biochemical Journal 270(3), 741-748 (1990)

52. Lee, Y.T., Lin, H.Y., Chan, Y.W.F., Li, K.H.C., To, O.T.L., Yan, B.P., Liu, T., Li, G., Wong, W.T., Keung, W., et al.: Mouse models of atherosclerosis: a historical perspective and recent advances. Lipids in Health and Disease 16(1), 12 (2017)

53. Lehoux, S., Lévy, B.I.: Collateral artery growth. Circulation Research; 99, 567-569 (2006)

54. Lehr, H., Hübner, C., Nolte, D., Finckh, B., Beisiegel, U., Kohlschütter, A., Messmer, K.: Oxidatively modified human low-density lipoprotein stimulates leukocyte adherence to the microvascular endothelium in vivo. Research in Experimental Medicine 191(1), 85-90 (1991)

55. Ley, K., Laudanna, C., Cybulsky, M.I., Nourshargh, S.: Getting to the site of inflammation: the leukocyte adhesion cascade updated. Nature Reviews Immunology 7 (9), 678-689 (2007)

56. Libby, P.: Inflammation in atherosclerosis. Nature 420, 868-874 (2002)

57. Lin, J., Li, H., Yang, M., Ren, J., Huang, Z., Han, F., Huang, J., Ma, J., Zhang, D., Zhang, Z., et al.: A role of RIP3-mediated macrophage necrosis in atherosclerosis development. Cell Reports 3(1), 200-210 (2013)

58. Liu, X., Fan, Y., Deng, X.: Effect of spiral flow on the transport of oxygen in the aorta: a numerical study. Annals of Biomedical Engineering 38(3), 917-926 (2010)

59. Mackness, M., Abbott, C., Arrol, S., Durrington, P.: The role of high-density lipoprotein and lipid-soluble antioxidant vitamins in inhibiting low-density lipoprotein oxidation. Biochemical Journal 294(3), 829-834 (1993) 
60. Maiellaro, K., Taylor, W.R.: The role of the adventitia in vascular inflammation. Cardiovascular Research 75(4), 640-648 (2007)

61. Maoz, H., Polliack, A., Barak, V., Yatziv, S., Biran, S., Giloh, H., Treves, A.J.: Parameters affecting the in vitro maturation of human monocytes to macrophages. Stem Cells 4(3), 167-185 (1986)

62. Melmed, R., Karanian, P., Berlin, R.: Control of cell volume in the J774 macrophage by microtubule disassembly and cyclic AMP. The Journal of Cell Biology 90(3), 761-768 (1981)

63. Meyer, G., Tedgui, A., et al.: Effects of pressure-induced stretch and convection on low-density lipoprotein and albumin uptake in the rabbit aortic wall. Circulation Research 79(3), 532-540 (1996)

64. Moireau, P., Xiao, N., Astorino, M., Figueroa, C.A., Chapelle, D., Taylor, C.A., Gerbeau, J.F.: External tissue support and fluid-structure simulation in blood flows. Biomechanics and Modeling in Mechanobiology 11(1-2), 1-18 (2012)

65. Moore, K.J., Tabas, I.: Macrophages in the pathogenesis of atherosclerosis. Cell 145(3), 341-355 (2011)

66. Mozaffarian, D., Benjamin, E.J., Go, A.S., Arnett, D.K., Blaha, M.J., Cushman, M., Das, S.R., de Ferranti, S., Després, J.P., Fullerton, H.J., et al.: Heart disease and stroke statistics - 2016 update. Circulation 133(4), e38-e360 (2016)

67. Nagano, Y., Arai, H., Kita, T.: High density lipoprotein loses its effect to stimulate efflux of cholesterol from foam cells after oxidative modification. Proceedings of the National Academy of Sciences 88(15), 6457-6461 (1991)

68. National Institutes of Health, National Heart, Lung and Blood Institute: ATP III guidelines at-a-glance quick desk reference. NIH publication (01-3305) (2001)

69. Olgac, U., Kurtcuoglu, V., Poulikakos, D.: Computational modeling of coupled bloodwall mass transport of LDL: effects of local wall shear stress. American Journal of Physiology-Heart and Circulatory Physiology 294(2), H909-H919 (2008)

70. Olgac, U., Poulikakos, D., Saur, S.C., Alkadhi, H., Kurtcuoglu, V.: Patient-specific three-dimensional simulation of LDL accumulation in a human left coronary artery in its healthy and atherosclerotic states. American Journal of Physiology-Heart and Circulatory Physiology 296(6), H1969-H1982 (2009)

71. Parton, A., McGilligan, V., O'Kane, M., Baldrick, F.R., Watterson, S.: Computational modelling of atherosclerosis. Briefings in Bioinformatics 17(4), 562-575 (2016)

72. Peiffer, V., Sherwin, S.J., Weinberg, P.D.: Does low and oscillatory wall shear stress correlate spatially with early atherosclerosis? A systematic review. Cardiovascular Research 99(2), 242-250 (2013)

73. Phillips, M.C., Gillotte, K.L., Haynes, M.P., Johnson, W.J., Lund-Katz, S., Rothblat, G.H.: Mechanisms of high density lipoprotein-mediated efflux of cholesterol from cell plasma membranes. Atherosclerosis 137, S13-S17 (1998)

74. Prosi, M., Zunino, P., Perktold, K., Quarteroni, A.: Mathematical and numerical models for transfer of low-density lipoproteins through the arterial walls: a new methodology for the model set up with applications to the study of disturbed lumenal flow. Journal of Biomechanics 38(4), 903-917 (2005)

75. Rajman, I., Eacho, P.I., Chowienczyk, P., Ritter, J.: LDL particle size: an important drug target? British Journal of Clinical Pharmacology 48(2), 125-133 (1999)

76. Rappitsch, G., Perktold, K.: Pulsatile albumin transport in large arteries: a numerical simulation study. Journal of Biomechanical Engineering 118, 511-519 (1996)

77. Resnick, N., Yahav, H., Shay-Salit, A., Shushy, M., Schubert, S., Zilberman, L.C.M. Wofovitz, E.: Fluid shear stress and the vascular endothelium: for better and for worse. Progress in Biophysics and Molecular Biology 81(3), 177-199 (2003)

78. Ross, R.: Atherosclerosis - an inflammatory disease. New England Journal of Medicine 340(2), 115-126 (1999)

79. Sherman, T.F.: On connecting large vessels to small. The meaning of Murray's law. The Journal of General Physiology 78(4), 431-453 (1981)

80. Shirai, T., Hilhorst, M., Harrison, D.G., Goronzy, J.J., Weyand, C.M.: Macrophages in vascular inflammation-from atherosclerosis to vasculitis. Autoimmunity 48(3), 139-151 (2015) 
81. Sill, H.W., Chang, Y.S., Artman, J.R., Frangos, J., Hollis, T., Tarbell, J.: Shear stress increases hydraulic conductivity of cultured endothelial monolayers. American Journal of Physiology-Heart and Circulatory Physiology 268(2), H535-H543 (1995)

82. Stary, H.C., Chandler, A.B., Dinsmore, R.E., Fuster, V., Glagov, S., Insull, W., Rosenfeld, M.E., Schwartz, C.J., Wagner, W.D., Wissler, R.W.: A definition of advanced types of atherosclerotic lesions and a histological classification of atherosclerosis. Circulation 92(5), 1355-1374 (1995)

83. Stender, S., Zilversmit, D.: Transfer of plasma lipoprotein components and of plasma proteins into aortas of cholesterol-fed rabbits. Molecular size as a determinant of plasma lipoprotein influx. Arteriosclerosis, Thrombosis, and Vascular Biology 1(1), 38-49 (1981)

84. Stocker, R., Keaney, J.F.: Role of oxidative modifications in atherosclerosis. Physiological Reviews 84(4), 1381-1478 (2004)

85. Sun, N., Wood, N.B., Hughes, A.D., Thom, S.A., Xu, X.Y.: Fluid-wall modelling of mass transfer in an axisymmetric stenosis: effects of shear-dependent transport properties Annals of Biomedical Engineering 34(7), 1119-1128 (2006)

86. Sun, N., Wood, N.B., Hughes, A.D., Thom, S.A.M., Xu, X.Y.: Influence of pulsatile flow on LDL transport in the arterial wall. Annals of Biomedical Engineering 35(10), 1782-90 (2007)

87. Suo, J., Ferrara, D.E., Sorescu, D., Guldberg, R.E., Taylor, W.R., Giddens, D.P.: Hemodynamic shear stresses in mouse aortas implications for atherogenesis. Arteriosclerosis, Thrombosis, and Vascular Biology 27(2), 346-351 (2007)

88. Tabas, I., Marathe, S., Keesler, G.A., Beatini, N., Shiratori, Y.: Evidence that the initial up-regulation of phosphatidylcholine biosynthesis in free cholesterol-loaded macrophages is an adaptive response that prevents cholesterol-induced cellular necrosis proposed role of an eventual failure of this response in foam cell necrosis in advanced atherosclerosis. Journal of Biological Chemistry 271(37), 22,773-22,781 (1996)

89. Tedgui, A., Lever, M.: Filtration through damaged and undamaged rabbit thoracic aorta. American Journal of Physiology - Heart and Circulatory Physiology 247(5), H784-H791 (1984)

90. Thomas, S.R., Mikulecky, D.C.: Transcapillary solute exchange: A comparison of the kedem-katchalsky convection-diffusion equations with the rigorous nonlinear equations for this special case. Microvascular Research 15(2), 207-220 (1978)

91. Thon, M.P.: Multiphysics modeling and quantification of early atherosclerosis. Ph.D thesis, Technische Universität München (2018)

92. Thon, M.P., Ford, H.Z., Gee, M.W., Myerscough, M.R.: A quantitative model of early atherosclerotic plaques parameterized using in vitro experiments. Bulletin of Mathematical Biology 80(1), 175-214 (2018)

93. Thon, M.P., Hemmler, A., Glinzer, A., Mayr, M., Wildgruber, M., Zernecke-Madsen, A. Gee, M.W.: A multiphysics approach for modeling early atherosclerosis. Biomechanics and Modeling in Mechanobiology 17(3), 617-644 (2018)

94. Tomaso, G.D., Díaz-Zuccarini, V., Pichardo-Almarza, C.: A multiscale model of atherosclerotic plaque formation at its early stage. IEEE Transactions on Biomedical Engineering 58(12), 3460-3463 (2011)

95. Tompkins, R.G.: Quantitative analysis of blood vessel permeability of squirrel monkeys. American Journal of Physiology - Heart and Circulatory Physiology 260(4), H1194H1204 (1991)

96. Véniant, M.M., Withycombe, S., Young, S.G.: Lipoprotein size and atherosclerosis susceptibility in Apoe-/- and Ldlr-/- mice. Arteriosclerosis, Thrombosis, and Vascular Biology 21(10), 1567-1570 (2001)

97. Wentzel, J.J., Chatzizisis, Y.S., Gijsen, F.J., Giannoglou, G.D., Feldman, C.L., Stone P.H.: Endothelial shear stress in the evolution of coronary atherosclerotic plaque and vascular remodelling: current understanding and remaining questions. Cardiovascular Research 96(2), 234-243 (2012)

98. Whitesall, S.E., Hoff, J.B., Vollmer, A.P., D'Alecy, L.G.: Comparison of simultaneous measurement of mouse systolic arterial blood pressure by radiotelemetry and tail-cuff methods. American Journal of Physiology-Heart and Circulatory Physiology 286(6), H2408-H2415 (2004) 
99. Whitman, S.C.: A practical approach to using mice in atherosclerosis research. The Clinical Biochemist Reviews 25(1), 81 (2004)

100. Williams, K.J., Tabas, I.: The response-to-retention hypothesis of early atherogenesis. Arteriosclerosis, Thrombosis, and Vascular Biology 15(5), 551-561 (1995)

101. Witztum, J.L., Steinberg, D.: Role of oxidized low density lipoprotein in atherogenesis Journal of Clinical Investigation 88(6), 1785 (1991)

102. World Healt Organisation (WHO): Cardiovascular diseases, fact sheet $\mathrm{n}^{\circ} 317(2017)$

103. Yang, N., Vafai, K.: Modeling of low-density lipoprotein (LDL) transport in the artery effects of hypertension. International Journal of Heat and Mass Transfer 49(5), 850-867 (2006)

104. Yao, P.M., Tabas, I.: Free cholesterol loading of macrophages induces apoptosis involving the fas pathway. Journal of Biological Chemistry 275(31), 23,807-23,813 (2000)

105. Zi, Z.: Sensitivity analysis approaches applied to systems biology models. IET Systems Biology 5(6), 336-346 (2011) 